\title{
Endstopping and Curvature
}

\author{
Allan C. Dobbins \\ B Sc . (Dathousie University), B. Eng . (McGill University)
}

\author{
Department of Electrical Engineering \\ McGill University
}

A thesis submitted to the Faculty of Graduate Studies and Research

in partial fulfillment of the requirements for the degree of

Master of Engineering

December 15th. 1988

(C) Allan C. Dobbins 


\section{Endstopping and Curvature}




\section{Abstract}

Hypercomplex or endstopped visual cortical neurons are usually supposed to be concerned with length or end point analysis. However, recent evidence demonstrates that endstopped neurons are curvature-selective, a connection that we explore here in some detail. A model of endstopped simple cells is developed and a variety of computational simulations examıne the connection of the model to the reported length and orientation responses of endstopped neurons. Even and odd versions of the model are described. both of which are shown to be curvature-selective. Even-symmetric instances of the model respond well to thın curves over a range of curve orientation and curvature, independent of sign of curvature. In contrast, odd-symmetric instances respond to both thin and thick curves while exhibiting a more complex curvature-sign dependence - responding in a signselective fashion to curved lines but not to curved edges Finally, the response of the endstopped model to curve singularities is explored. and the possible role of nonendstopped and endstopped cells in building curve descriptions is discussed. 


\section{Résumé}

Les neurones hypercomplexes ou inhibé en bout (endstopped) du cortex visuel sont ordinaırement reliées à l'analyse des longeurs ou des extrémités. Pourtant, des résultats récents démontrent que les neurones inhibé en bout sont sélectıfs à la courbures. ce qui est exploré ici. Un modèle de cellules simples inhıbé en bout est developpé et une ensemble de simulations par ordınateur sont utilisées pour examıner le lien entre le modèle et les réionses observées des neurones inhibé en bout aux longeurs et aux orientations. Des versions paires et impaires du modeles sont décrites. chacune étant démontrée selective à la courbure. Les versions paire-symmetriques du modèle répondent bıen à des courbes fines dans une gamme d'orientations et de courbures, et ce. indépendanment du signe de la courbure Au contraıre. les versıons impaıres-symmetriques répondent à des courbes autant fines que grasses, tout en présentant une dépendance plus complexe envers le sıgne de la courbure - elles répondent d'une manière sélective au signe pour des lignes courbes. mais d'une manière non sélective pour des bordures courbes Finalement. la réponse des modèles inhibé en bout aux singularités de courbes est explorée. et le rôle possıble des cellules inhibé en bout et non inhibé en bout dans la description de courbes est discuté 
Acknowledgements

I would like to thank all those who have made the present work possible. In particular. I would like to thank my friend Lee Iverson for many hours of stimulating discussion and programming wizardry in building tools for the Symbolics LISPM environment on which this work depends. I must also acknowledge the Influence of Kama Gupta and Benjamin Kimia. and all the other denizens of MCRCIM for collectively creating such a pulsing. thriving environment Lastly I wish to thank Professors Steven Zucker and Max Cynader for support and influences inestimable.

iv 


\section{Contents}

Chapter 1 Introduction. $\ldots \begin{array}{lllllll} & \ldots & \ldots & \ldots & \ldots & \ldots & \ldots\end{array}$

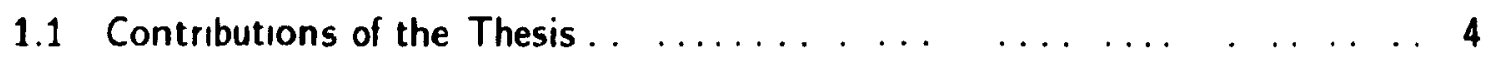

1.2 Organization of the Thesis $\begin{array}{lllllllll}\ldots & \ldots & \ldots & \ldots & \ldots & \ldots & \ldots & \ldots & 5\end{array}$

Chapter 2 Historical Background $\ldots \ldots \ldots \ldots \ldots \ldots$

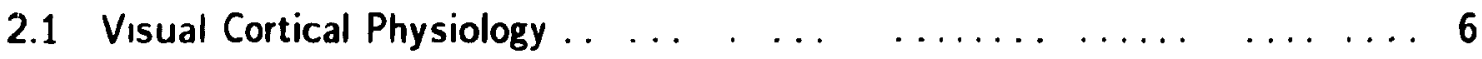

2.2 Psychophysics and Curvature $\ldots \ldots \ldots \ldots \ldots \ldots, \ldots \ldots \ldots$

Chapter 3 The Endstopped Simple Cell Model.... . ... . . . . . . 9

3.1 Formulation of the Model. $\ldots \ldots \ldots \ldots \ldots \ldots \ldots \ldots \ldots \ldots$

3.2 Methods.. ... ................................. 12

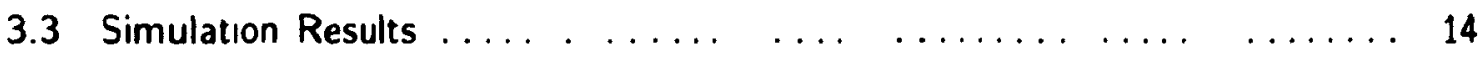

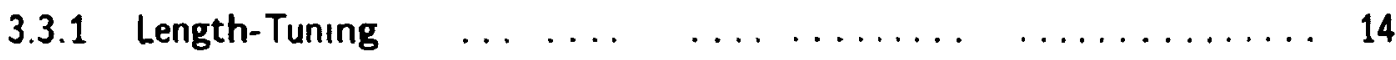

3.3.2 Orientation-Tuning $\ldots \ldots \ldots \ldots \ldots \ldots \ldots \ldots \ldots \ldots \ldots \ldots$

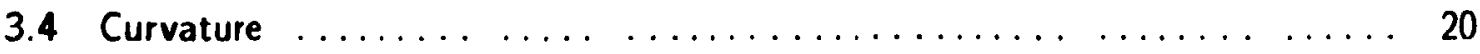

3.4.1 Differential Geometry and Endstopping............... 20

3.4.2 Response of the ES Model to Curved Lines and Edges..... . . . . 20

3.5 Response to Discontinuities and Singularities .............. 25

3.5.1 Response to Chevrons......................... 26

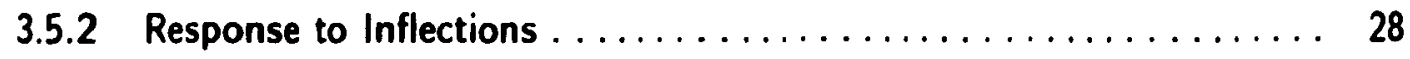

Chapter 4 Discussion $\ldots \ldots \ldots \ldots \ldots \ldots \ldots \ldots \ldots \ldots \ldots \ldots \ldots \ldots \ldots \ldots \ldots$

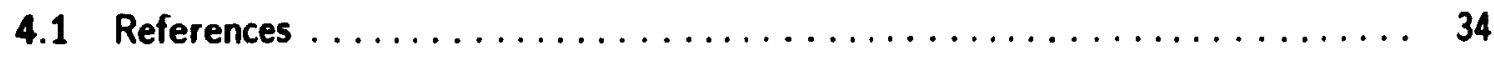

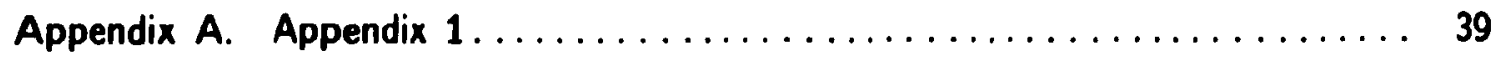


The visible world consists of surfaces and objects on surfaces (Gibson. 1951) Closed bodies also present surfaces but they are generally bounded by visible contours or curves Surfaces may give rise to contours alor.g ridges. cracks and folds. or where an outcropping occludes the matter behind it. All of these contours may be important in providing information about the shape of objects and surfaces

To describe the shape of a contour one might catalog the values of some property of the curve The natural choice is curvature and both extrema of curvature and the locus of inflections (zeros) have been used Attneave (1954) demonstrated the significance of the high curvature information in pictures by modifyıng a line drawing of a cat by connectıng the curvature maxima by straight lines Remarkably. the picture is still immediately recognizable as a cat and looks virtually as good as the original. Leyton (1988) proposed a shape grammar based on the sequence of curvature extrema encountered in traversing a smooth. closed curve. Based on the concept of transversality from differential topology. Hoffman and Richards (1985) have proposed that shapes be decomposed into parts at the minima of negative curvature. Asada and Brady (1986) attempt to infer the shape of an object from the sequence of curvature extrema and inflections on its bounding contour. It has also been argued that curvature is essential to reliably find the contours in images (Parent \& Zucker, 1985). It is in the latter context that the present work has developed.

Curve inference in visual images differs from curve-fitting (approximating or interpolating a curve to given points) in important respects. First. image contours differ 
from their ideal mathematical counterparts in having variable width. Intensity. and in being discrete Second. multiple curves may be present in a variety of intersectıng. occluding and apposing relations with one another Third, there are points that belong to no curve (noise). Therefore the problem of curve inference can be described as determınıng the par:ition of points among the curves in an image and their ordering within each curve The inference process must locally estımate the geometric structure of the scene determining where change is smooth and where it is abrupt. and hence over what spatial range a particular approximant is valid An abstract formulation of the problem is as follows.

A curve through points $\mathbf{A}$ and $\mathbf{B}$ exists if and only if:

1. The geometric estımates at $\mathbf{A}$ and $\mathbf{B}$ are mutually consistent, and

2. The geometric estimates at all points that are interpolants from $\mathbf{A}$ and $\mathbf{B}$ are mutually consistent

If $\mathbf{A}$ and $\mathbf{B}$ are far apart, it is necessary that the geometric estımates be of high order (many terms in the Taylor series. see Appendix 1). otherwise there are many possible curves that satisfy the boundary conditions. The approach of Parent and Zucker (1985) involved making dense estımates of the local geometric structure (tangent and curvature) and enforcing a simple consistency relation (co-circularity) over them (Figure 1.1) The two estımates are consistent only if each falls within the other's bounds. Hence, the recursive decomposition is collapsed into a local, parallel procedure.

The tangent points in the direction in which the curve is moving and curvature is the rate of change of the tangent direction. Visual cortical neurons exhibit marked orientation preference and can be viewed as tangent estimators. Consider the expected response of a cortical simple cell to a curved arc centered on the RF and with its tangent parallel to the cell's preferred orientation The response is weak to a highly curved arc but increases with the radius of curvature. Changing the size of the RF changes the rate at 


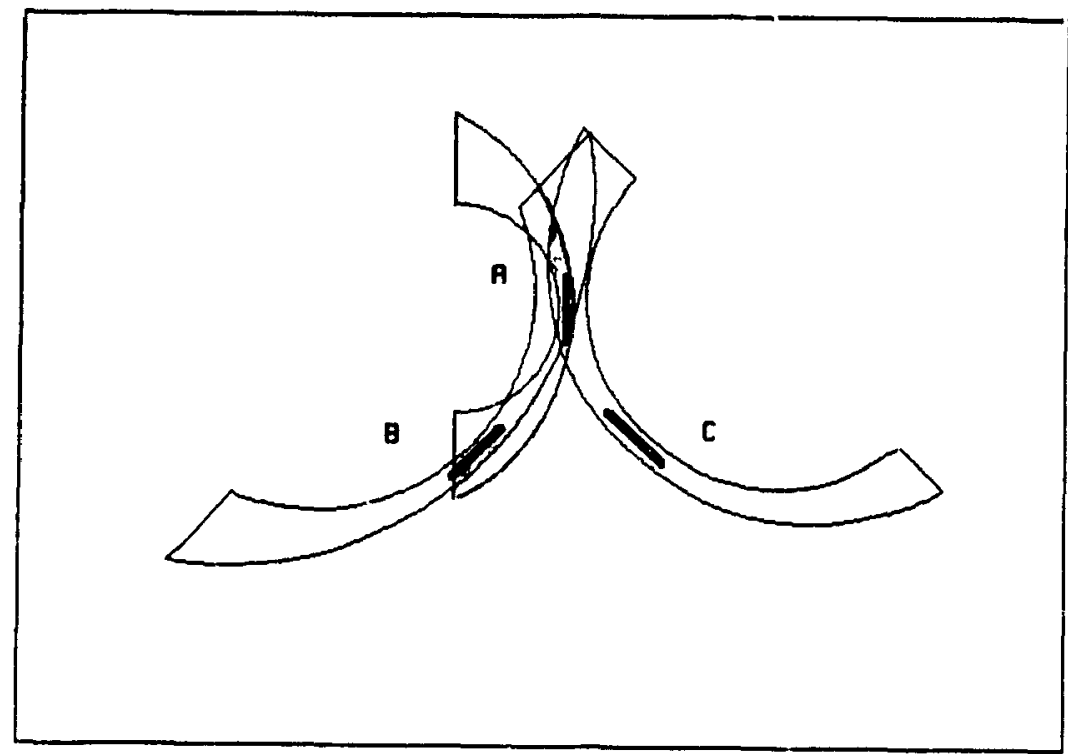

Figure 1.1 The arcs that contain the tangent estimates at $\mathbf{A}, \mathbf{B}$, and $\mathbf{C}$ represent bounds on the curvature estimates at those positions Two tangents are co-circular

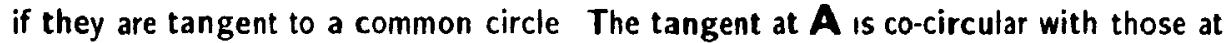
$\mathbf{B}$ and $\mathbf{C}$ within the quantization of position. orientation and curvature Estimates of magnitude and direction of curvature supply additional constraints The tangent at $\mathbf{A}$ is consistent with the curvature estimate at $\mathbf{C}$ since it is contained withın its bounds and is co-circular Notice that the sign of curvature information is not compatible with a common curcle joining $\mathbf{A}$ and $\mathbf{C}$ However the estimates at $\mathbf{A}$ and B are curvature-consistent Two nearby estimates are curvature-consistent if each includes the sther within its curvature bounds - graphically. if the intersection of the two sets of arcs (shaded region) contains both points

which the response grows with radius. Therefore RFs of different size provide information about the way in which a curve departs from its tangent line (curvature).

Endstopped or hypercomplex neurons were first identified by Hubel and Wiesel (1965), and exhibit ronmonotonic length-response. The simplest model that accounts for this observation has inhibitory endzones synthesized from a large RF neuron Such a cell should recpond in a curvature-selective fashion. The endstopped (ES) neuron model is constructed by taking the "difference" of aligned RFs of different size:

$$
R_{E S}=\Phi\left(c_{S} \cdot \Phi\left(R_{S}\right)-c_{L} \cdot \Phi\left(R_{L}\right)\right)
$$

small (S) and large (L) RFs may be simple or complex, and, if simple. may be even or odd. We model the response of such cells as spatial convolutions against the image $\left(R_{S}, R_{L}\right.$; 
$\left.R_{\imath}=k * I\right)$. Each convolution result is passed through a function $(\Phi(\cdot))$ which models the inability of neurons to represent negative values on a low spontaneous firing baseline. Scalar constants $c_{S}$ and $c_{L}$ are introduced to balance the responses between $S$ and $L$. The circuitry underlying the model is depicted in Figure 1.2.

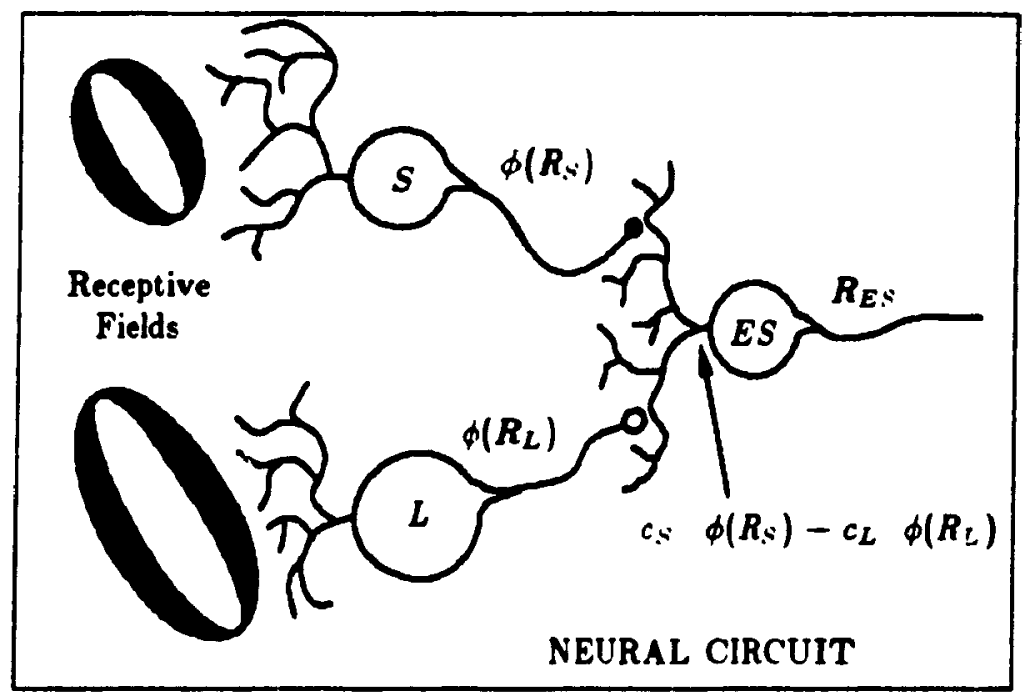

Figure 1.2 A schematic model of the endstopped circuit In the example shown both small (S) and large (L) RF components are simple cells and provide excitation and inhibition. respectively. to the endstopped (ES) simple cell upon which they converge

Provided with the local tangent and curvature estimates, it should be possible to locate gaps and singularities. and to synthesize smooth approximants that satisfy the local geometric constraints while avoiding inappropriate smoothing across distinct structures. Networks to perform these computations are developed elsewhere (Parent \& Zucker, 1985: Iverson. 1988). The purpose of the present work is to undertake an examination of the relation between endstopping and curvature to examine how much of curve inference can be attributed to the receptive fields of single neurons and how much must be assigned to network interactions between those cells - the projective fields of neurons.

\subsection{Contributions of the Thesis}

My specific contributions are: 
- The idea that endstopped neurons are estimators of curvature.

- Developing a model of endstopped simple cells that accounts for their common properties and which also exhibits curvature-selectivity.

- Explicating how receptive field symmetry determines curvature sign selectivity.

- Exploration of the response of the model to curve singularites and discontinuities has suggested a framework for understanding the role of visual cortical neurons in representing the local structure of curves.

\subsection{Organization of the Thesis}

Chapter 2 contains a review of the background to the present work and Chapter 3 contains the core of the thesis. developing the model and describing the results of computational experiments. Chapter 4 discusses and summarizes the ideas comprising this work.

Parts of this thesis and extensions of it are currently in the process of being published as research articles with the coauthorship of my advisors Prof. Steven Zucker and Prof. Max Cynader. 


\section{Chapter 2}

\section{Historical Background}

\subsection{Visual Cortical Physiology}

Visual cortical neurons are considerably more specific in their responses than their antecedents in the pathway from retina to primary visual cortex. Cells are described as simple or complex according to a number of criteria. the most sigificant of which is whether the ON and OFF regions parallel to the cell's preferred orientation are separated or not. respectively (Hubel \& Wiesel, 1962). Along the long axis of the receptive field (RF hereaftes) cells also differ in their length summation characteristics. Nonendstopped cells exhibit monotonic response with increasing length. In contrast. endstopped cells are nonmonotonic. with response increasing up to some length and then decreasing. often to an asymptote (Hubel \& Wiesel. 1965). An issue of contention has been whether there is a continuum between these two types.

Although Hubel and Wiesel (1965) recognized that endstopped cells could be expected to respond to corners and curves and show the response of a number of cells to corners, this insight has been largely ignored by subsequent researchers. Hubel and Wiesel described lower order hypercomplex cells in Areas 18 and 19 of the cat as being either singly or doubly-stopped depending on whether inhibition was present at one or both ends. Subsequent investigations also find endstopping in cat striate cortex (Dreher. 1972: Kose. 1977: Kato et al. 1978). and report the striate cells to have approximately balanced double stopping (Orban et al, 1979a). In this study. we model only double stopped cells. 
2 Historical Background

Careful examination of the inhibitory "endzones" of endstopped RFs has revealed that they are orientation selective and tuned to the same orientation as the RF center (Hubel \& Wiesel, 1965: Orban, et al, 1979b): that the most sensitive region of the endzone is approxımately collinear with the most sensitive region of the RF center (Orban et al. 1979b): and that the end zones partly or completely overlap with the RF center (Orban et al. 1979b) In the last few years, evidence both anatomical and physiological has suggested that endstopped cells may be built from combinations of nonendstopped cells (McGuire et al. 1984: Bolz and Gılbert. 1986). It is known that Layer VI contains long RF neurons (Gilbert. 1977), and more recently it has been shown that reversibly inactivasing Layer VI reduces or abolishes endstopping in the superficial layers (Bolz and Gilbert. 1986) suggesting an important role for the Layer $\mathrm{VI}$ cells in generating end inhibition These data are from the cat: the situation in the primate has been studied less. Recently we have reported that endstopped neurons exhibit curvature-selective responses that contrast with the response to curves exhibited by nonendstopped cells (Dobbıns et al. 1987). Previously. only two groups had examined the response of cortical cells to curves The first of these investigations examined only nonendstopped cells (Heggelund \& Hohmann. 1975), and Hammond and Andrews (1978) restricted their stimuli to avoid RF endzones so virtually all striate cortical cells preferred straight lines to chevrons.

Our model is based on two observations. 1. curvature can be viewed as deviation form straightness, and 2. a measure of this deviation can be obtained from multiple orientation-selective cells. In particular, since simple $\mathrm{KFs}$ have an oriented structure that respond best to straight contours. RFs of the same central position (center of mass) and orientation but of different size together provide information about the deviation from straightness around that position. This provides a formal interpretation to one of the candidate models for synthesizing endstopped neurons (Hubel \& Wiesel, 1965: Orban et al. 1979b). The same argument holds for the subset of complex cells that exhibit linear length summation. In this thesis attention is focused on neurons synthesized from simple components. but elsewhere we examine endstopped models with one or more complex components (Dobbins et al. 1988). 


\subsection{Psychophysics and Curvature}

Since the demonstration by Attneave (1954) of the significance of curvature information. a large number of psychophysical studies have examined curvature. The first one of import to the present study is the attempt of Blakemore and Over (1974) to determine if the human visual system contains curvature detectors. They found that it was possible to elicit curvature-selective threshold elevation, but concluded that the results could not be accounted for by local curvature detectors. and favoured the interpretation of adaptation of local orientation detectors.

Timney and McDonald (1978) employed curved gratings and also found adaptation selective for both the magnitude and sign of curvature. In addition. control experiments revealed that. although the adaptation effect depended critically upon the orientation of the test stimulus, the effect was not an orientation adaptation effect. Because the curvature adaptation was orientation-dependent. these investigators also favoured an explanation in terms of orientation detectors. In both the experiments of Blakemore and Over (1974) and Timney and McDonald (1978) curvature was conceived as independent of orientation. If. on the other hand. curvature is estimated independently at each orientation (by neurons with RFs at the same position and orientation) as we propose endstopped neurons do. then one would expect the results obtained by Timney and McDonald (1978).

In the next chapter the model is elaborated and simulations based on it are described. 


\subsection{Formulation of the Model}

To model endstopping we focus on the spatial properties of simple type RFs. Binocularity is not incorporated and temporal properties are collapsed. allowing the response of a simple cell to be treated as a spatial convolution. From time to time we shall comment on the plausibility of these simplifications in connection with specific results.

The endstopped simple model is obtained by taking the difference of the response of two simple RFs at the same position and orientation. but of different size. Put differently, excitatory influence from the small RF cell and inhibitory influence from the large RF cell converge on the endstopped cell. Let $R_{S}$ denote the response of the small simple cell and $R_{L}$ the response from the large one. It is generally observed that Area 17 has low spontaneous activity. More specifically, data in Gilbert (1977) show that Layer VI cells exhibit very low spontaneous firing. and Kato et al (1978) report that endstopped simple cells in particular have virtually none. This corresponds to an inability to encode responses lower than the base frequency, and so. in the model, each response is half-wave rectified. Therefore, if $\phi(\cdot)$ is a rectifying or clipping function, which equals its argument when positive and is zero otherwise.

$$
\phi(x)= \begin{cases}x, & \text { if } x>0 \\ 0, & \text { otherwise. }\end{cases}
$$


the end-inhibited simple cell (ES) response is given by:

$$
R_{E S}=\phi\left(c_{S} \cdot \phi\left(R_{S}\right)-c_{L} \cdot \phi\left(R_{L}\right)\right)
$$

where $c_{S}$ and $c_{L}$ are positive constants that control the gan of the two components. Appendix 1 describes how varying the relative gain affects the behaviour of the model. In response to sinusoidal stimulı of varying phase $\phi\left(R_{S}\right)$ and $\phi\left(R_{L}\right)$ exhibit the half-wave rectified sinusoidal responses characteristic of simple cells (Movshon et al, 1978). Half-wave rectification increases the stimulus-specificity of the model by preventing a negative value for $R_{L}$ (caused by a net inhibitory stimulus) from being inverted into a positive contribution to $R_{E S}$, the response of the ES cell. Physiologically this means that hyperpolarization of the large inhibitory cell does not contribute to excitation of the endstopped cell.

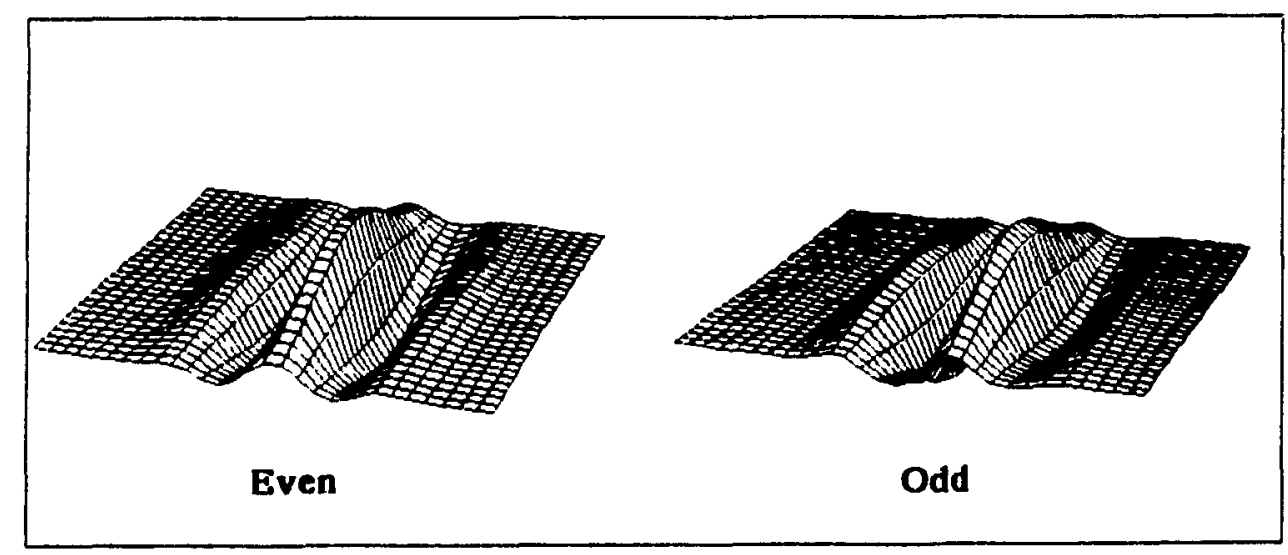

Figure 3.1 A graphical depiction of even and odd simple cell RFs (a) An even RF modeled by an elongated DOG. (b). An odd RF modeled by a Gabor function

The spatial RFs of simple cells come in even. odd and intermediate varieties (Heggelund. 1986; Jones \& Palmer, 1987a), and can be mathematically described in several different ways - as Differences of Gaussians (DOGs). differences of DOGs (Hawken \& Parker. 1987). Gabor functions (Marcelja. 1980). and as derivatives of Gaussians (Koenderink \& van Doorn. 1987) to name but a few. Each formulation has its advantages in simplicity or ability to account for the data (see Hawken \& Parker. 1987; Jones \& Palmer. 
1987b: Parker \& Hawken. 1988). In our simulations we have used both DOG and Gabor RF descriptions. Treating cell response as a spatial convolution against an image $I$, the kernel $k$ is the RF profile of the cell. Hence. the response $R$ is given by:

$$
R\left(x_{0}, y_{0}\right)=I \oslash k=\iint_{D=\Omega^{2}} I(\alpha, \jmath) \cdot k\left(x_{1,}-\alpha, y_{\prime \prime}-, 3\right) d a d s
$$

where $\left(x_{0}, y_{0}\right)$ fixes the RF position. Integration is limited to the region $D$ which represents the spatial extent of the RF

The elongated DOG description of even-symmetric simple RFs is obtained by taking the difference of two 2-D Gaussians of the same length but different width. Each Gaussian is normalized. A 2-D separable Gaussian with principal axes $x$ and $y$ is characterized by the quadruple $\left(\mu_{\tau}, \mu_{y}, \sigma_{x}, \sigma_{y}\right)$. Ignoring the position coordinates $\left(\mu_{x}=x_{0} . \mu_{y}=\right.$ $\left.y_{0}\right)$. the Gaussian is described as:

$$
G\left(\sigma_{x}, \sigma_{y}\right)=\frac{1}{2 \pi \sigma_{x} \sigma_{y}} e^{-\frac{1}{2}\left(\frac{x^{2}}{\sigma_{x}^{2}}+\frac{y^{2}}{\sigma_{y}^{2}}\right)}
$$

To describe the RF parameters consider a local coordinate system $(x, y)$ in which the preferred orientation aligns with $y$. For a DOG the RF shape may be usefully characterized by the following parameters. The length or size $S$ is approximately $4 \sigma_{y}$. the width ratio (WR) is $\sigma_{x 2} / \sigma_{x 1}$ and the single lobe or subunit aspect ratio (AR) is $\sigma_{y} / \sigma_{x 1}$. Hence the convolution kernel $k$ can be characterized as:

$$
k(x, y: S, A R, W R)=G_{1}\left(\mu_{x}, \mu_{y} ; \sigma_{x 1}, \sigma_{y}\right)-G_{2}\left(\mu_{x}, \mu_{y}: \sigma_{x 2}, \sigma_{y}\right)
$$

Intuitively, the spatial frequency response (in the usual sense) is determined by $\sigma_{x 1}, \sigma_{x 2}$ and their ratio, and the orientation tuning is determined by AR. Figure 3.1a depicts a cortical 
RF modeled as an elongated DOG. Note that the envelope of the RF has an aspect ratio given by $. A R \cdot{ }^{\prime} W^{\prime} R=\sigma_{y}, \sigma_{x 2}$ and hence the values $A R=5$. WR $=2.5$ used for the large cornponent correspond to an RF aspect ratio of 2.

When the simple RF is modeled as a Gabor function (a 2-D Gaussian modulated by a sinusoid). the following parameters are used. The size (S) is given by $4 \sigma_{4}$, the aspect ratio is $\sigma_{4}, \sigma_{x}$. the reference period $(P)$ of the sinusoid is given by $4 \sigma_{x}$, and the period ratio $(P R)$ is a multiplier of the reference period that gives the actual period Hence, in terms of these parameters, the radian frequency of the sinusord is given by $\omega=2 \pi, P \cdot P R$. For convenience the convolution kernel $k$ can be described as.

$$
k(x, y: S, A R, P R)=G\left(\mu_{x}, \mu_{y}: \sigma_{x}, \sigma_{y}\right) \sin (\iota \cdot x)
$$

Naturally. sines and cosines produce odd and even symmetry. respectively. Although intermediate phases are observed in cortical simple cells we model only those that are odd or even. An odd-symmetric RF represented by a Gabor function is shown in Figure 3.1b.

Several issues arise in specifying the exact form of the RFs These include the relationship between the spatıal frequency and orientation responses of the small and large components, and the implications for the curvature-response of ES cells. Since these issues are significant in their own right. we summarize them in Appendix 1 and treat them fully in another paper (Dobbins et al. 1988). For the remainder of this paper we use parameter values based on considerations discussed there, and that represent compromises between matched orientation tuning and spatial frequency response.

\subsection{Methods}

A simulation system was developed in LISP on a Symbolics 3670 computer. The model was evaluated using experiments analogous to those used in single cell neurophysiology: that is, an instance of the model was convolved against images of lines and 
edges of various lengths and widths at various positions and orientations with respect to the RF. In addition. curved lines and edges were tested over a range of radii, positions and orientations. The stimuli were etther binary-valued curves with a jagged appearance. or gray-level curves ( $8 \mathrm{bit})$ with a smooth appearance created using a supersampling technıque. In this technique the intensity value of a pixel is determined by considering the fraction of coverage of the pixel (treated as an 8 by 8 grid) by a contınuous curve

The previous section describes integration of continuous variables over a continous domain. The sımulatıons, in contrast, involve summation of finite precisıon variables over a discrete domain. Simple cell RFs were represented as 2-D arrays of double precision floating point numbers. The array elements were the samples of the continuous RF lying on a rectangular grid. Note that retınal sampling resembles perturbed hexagonal sampling and not the rectanguliar scheme used here The differences between the two are greatest for very coarse sampling Under the assumption that the continuous models are reasonable approximate descriptıons of RFs. fine sampling was employed to minımize the difference in numerical results between rectangular-sampled and continuous RFs. That is. RFs were represented by large arrays e.g. 60 by 60 elements. Representations of Gaussians were truncated at $=2 \sigma$

In electrophysiological experıments stimuli either move, or are stationary (flashed on and off). or are reversed in phase. The simulation experiments correspond most closely to the response of a cortical neuron to a stationary presentation of the stimulus. In this paper, line stimuli are chosen to be less than or equal to the excitatory width of the RF and, except where stated otherwise, are centered upon it. Edge stimuli are positioned at the boundary between antagonistic RF regions except where a contrary statement is made. We concentrated on three different classes of experiment

(1) Length Tuning. In these experiments, response to an optimally-oriented and positioned light bar is computed, as length is added symmetrically to each end of the bar. For the ood-symmetric model. the stimulus was centered on the peak of the positive lobe of the RF. 
(2) Orientation Tuning. To evaluate the orientation response of the model, a simulated light bar is positioned over the central RF and rotated in equal steps about its center.

(3) Curvature Tuning. Curvature response is evaluated by computing the response to semi-circular arcs of varying radius arranged so that the mid-point of the arc falls on the central RF and oriented in such a way that the tangent at mid-arc corresponds to the long axis of the RF of the cell The curves were either thin lines or curved edges In some experiments the curves are discontinuous in the tangent (chevrons) and in others inflected (curvature passes through zero).

\subsection{Simulation Results}

In the rest of the paper the response of instances of the model is evaluated with various contrast patterns. For those experiments that correspond to common visual cortical methodology results are compared to the characteristics of endstopped cells. while for novel stimuli the results predict particular responses from endstopped neurons. The choice of parameter values used is described in Appendix 1.

\subsubsection{Length-Tuning}

The endstopped property is defined in terms of the response of cortical cells to properly oriented line or edge stimuli of various lengths. The length-tuning curves that result are obtained either with stimuli moving in the preferred direction or with flashed stimuli positioned over the most sensitive portion of the RF. The spatial model of the RF used here can be thought of as collapsing the temporal dimension and hence the convolution value is assumed to correspond to something like total spikes in response to the stimulus. However, since most length-tuning data in the literature are obtained with moving stimuli it is important to know that the length-tuning curve is similar whether obtained with moving or stationary stimuli (Orban et al 1979a). implying that essential temporal interactions do 


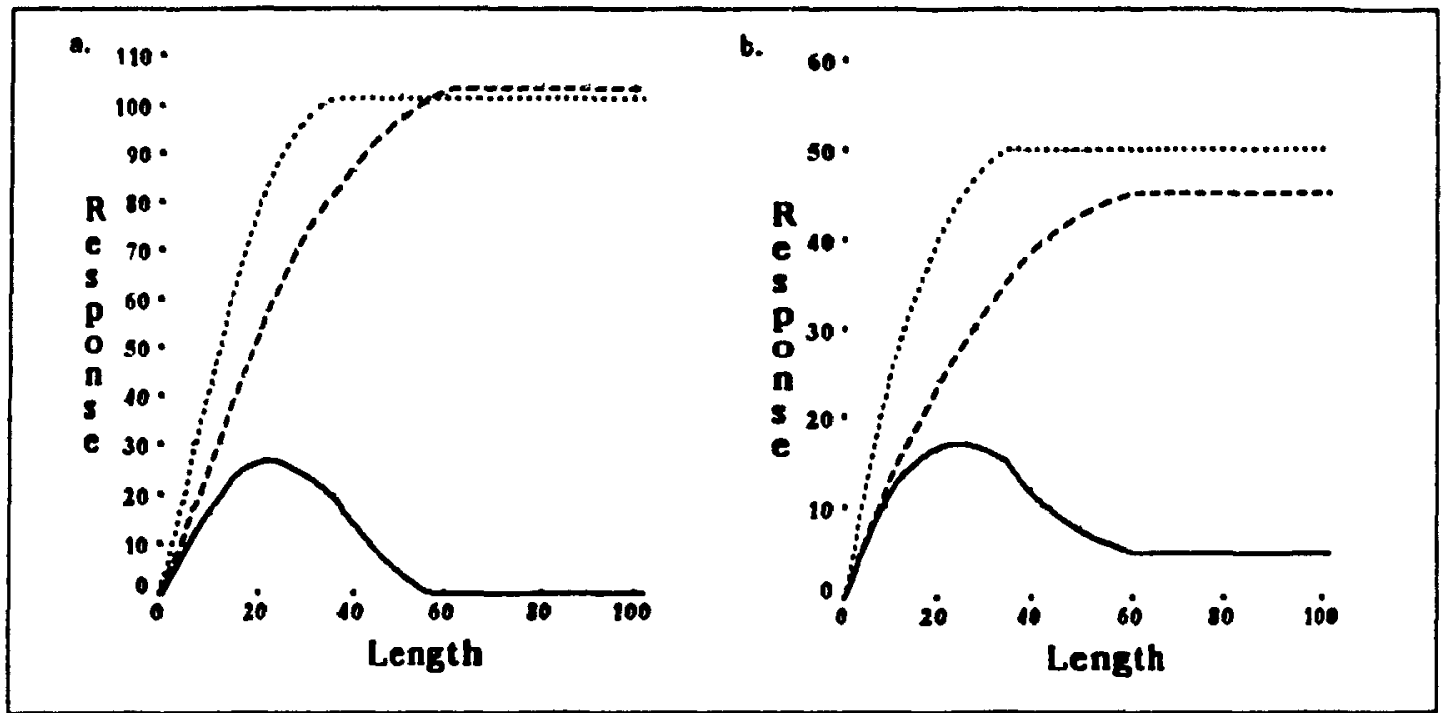

Figure 3.2 Length-tuning curves for even and odd RF ES cells and their components in each case the solid curve represents the ES cell. and the dotted and dashed curves the small and large components. respectively The even ES model (a) has components with lengths 35 and 61 pixels. weights of 18 and 1 ARs of 4 and 5 and each has a WR of 25 The components of the odd-symmetric instance have lengths of 34 and 60 pixels, weights of 17 and 1. ARs of 25 and 3. and PRs of 15

not distınguish the two cases, and therefore that the use of spatial convolutions is not a priori unreasonable.

Figure 3.2 shows the length-tuning for even and odd instances of the endstopped simple model. The responses of the even-symmetric version and its simple components are shown in Figure 3.2a. while the corresponding odd-symmetric data are shown in Figure 3.2b. In both cases. the difference in growth rate of the response of the small and large components produces the characteristic endstopped length-tuning curve. By changing the relative size or weight of the simple components one obtains curves with different peaks. excitatory widths, and asymptotic values. Note that in both cases the peak of the lengthtuning curve is significantly shorter than the length of the short excitatory RF. a point which is returned to in the next section

\subsubsection{Orientation-Tuning}

The response of even- and odd-symmetric versions of the model to lines of various orientations is shown in Figure 3.3. Figures 3.3a,b depict the orientation-tuning curve 
-.

a. 110

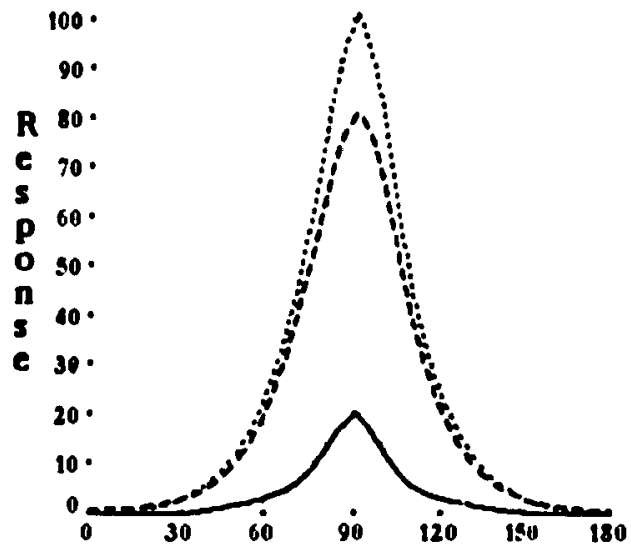

c.

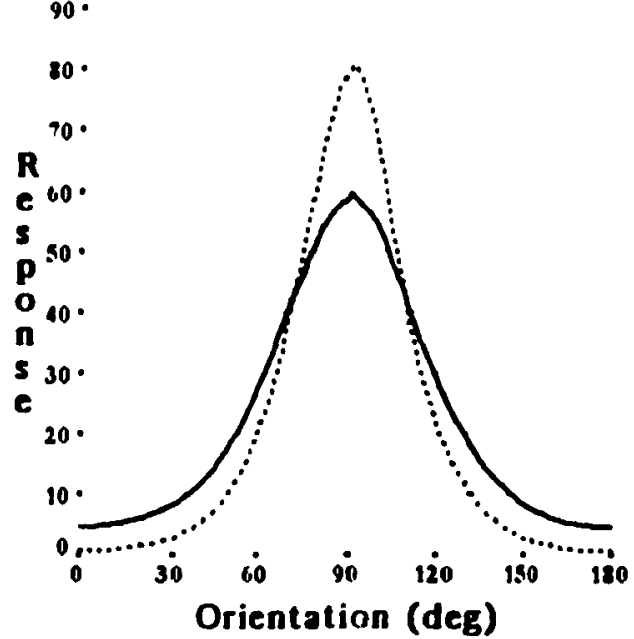

b. $60^{\circ}$

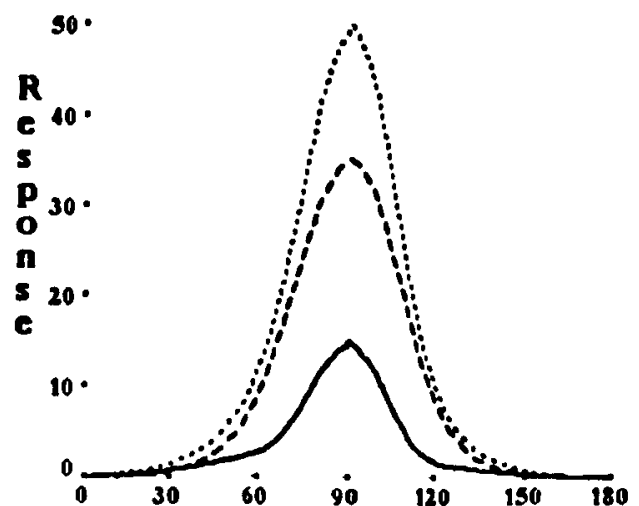

d.

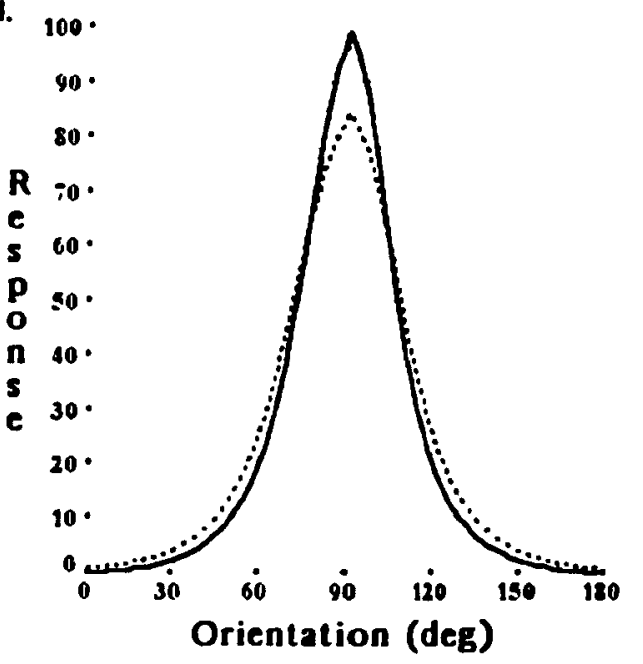

Figure 3.3 Orientation-tuning curves for the even (a) and odd (b) RF ES instances introduced in Figure 32 A bar equal in length to the short component RF and 3 pixels wide is convolved with the RF at 90 equally spaced orientations The graphs show the short component (dotted). long component (dashed). and endstopped instance (solid) In $b$ the line rotates about the peak of the positive lobe of the RF (c) Orientation response of the short even simple component to bars of length 23 (solid) and 35 pixels (dotted) (d) Response of the large even component to the same stimuli (same convention as (c)). In both cases the tuning is wider for the shorter bar. but the change is greater for the short RF

for the even instance and components, and the odd instance and components. respectively. In both cases, the measurements were made with stimuli equal in length to the small simple RF. The half-width at half-height of the even orientation-tuning curve is approximately 10 degrees. while it is approximately 15 degrees for the odd ES curve. The small and large components of the even-symmetric instance in Figure 3.2a yield figures of 17 and 20 
degrees, respectively. These numbers correspond to narrowly-tuned cortical units In the sample of endstopped sımple cells obtained by Kato et al (1978). the mean and standard deviation of response half width at half height were $22.5=12.9$ degrees, while the values for sımple cells were $17.1=51$. a value largely in accord with other studies (for a summary of orientation-tunıng studies consult Orban (1984)) The orientation-tunıng width depends on both the length and width of the test stımulus, tunıng becoming narrower when either parameter increases Because of the greater aspect ratıo of the large components in both the even and odd instances. one would expect them to exhıbit narrower orientation-tunıng than the small components However the stimulus used is chosen for its correspondence to the dimensions of the small simple RFs in both cases. Therefore, the large simple cells respond over a broader range than they would to a larger stımulus

The implications for the orientation-tuning of endstopped cells are twofold, and they push in opposite directions. First. since the cell responds over a broader range of orientations to the short stimulus, it produces inhibition over a broader range of orientations and hence narrows the range of orientations over which the endstopped cell responds This can be observed in Figure 3.3a and $b$. The second factor however is that the peak of the length-tuning curve for the ES cell model. and almost certainly for endstopped neurons as well. is less than the length of the short excitatory RF This follows from the overlap of the end zones with the excitatory region The peak occurs at that length at which an incremental increase causes more inhibition than excitation to be added to the response of the endstopped cell. For some of the RF formulations it is possible to explicitly solve this. Depending, roughly speaking. on the size and weights of the simple components. the peak of the length-response may be as little as 60 percent of the length of the small excitatory RF length. If the orientation-tuning curve were measured with a stimulus of this length, the small excitatory cell would respond over a broader range than to a full length stimulus. Hence. the two effects work against each other, the first tending to narrow the measured orientation response of the endstopped cell. the second tending to broaden it. Figures $3.3 \mathrm{c}$ and $3.3 \mathrm{~d}$ display the response of the small and large components of the even instance to stimuli of length 23 and 35 units (the peak of the length-tuning curve, and 
the length of the short RF. respectively). The change in response amplitude and width is greater for the small component than for the large one, causing the half width at half height of the endstopped cell to increase when the shorter stimulus is used. In general, one would expect the width of orientation-tuning of endstopped simple cells to be overestimated since the interrogating stimuli are shorter in relation to the excitatory RF than those used with sımple cells

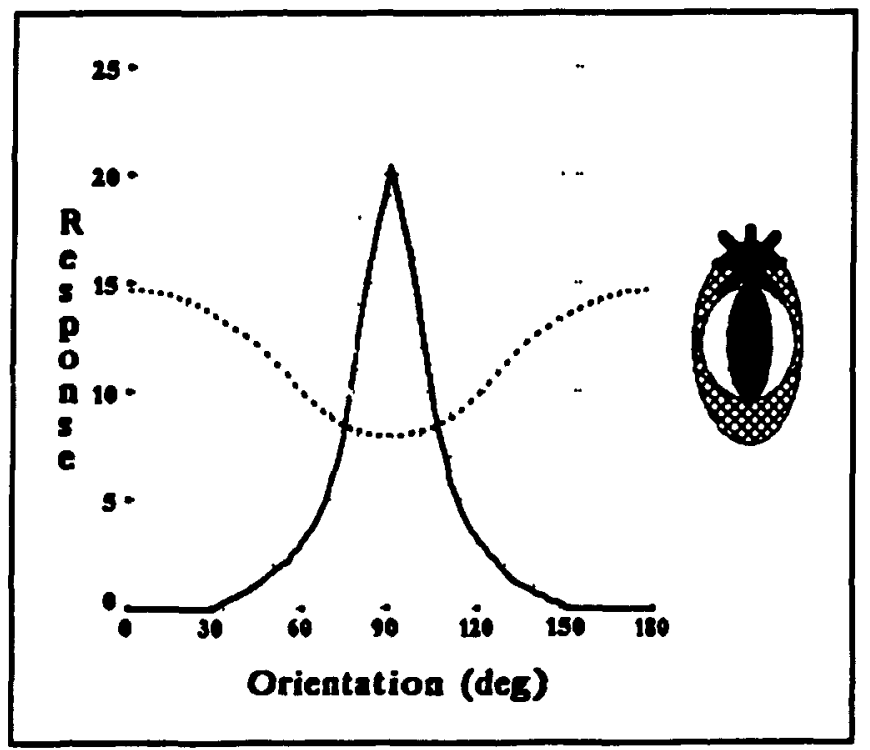

Figure 3.4 Response of the even ES instance to an optimally-oriented central bar 35 pixels long and a variable orientation bar 13 pixels long in one end zone Both bars are 3 pixels wide The solid curve repeats the orientation curve from Figure 33 a and the dotted curve shows the effect of the bar in the endzone on the response of the central bar Inhibition 15 most effective at the optimal orientation of the center.

Just as orientation-tuning depends on stimilus length, length-tuning measured at the wrong orientation causes misclassification and mismeasurement (Orban et al.. 1979a). The model captures both these: nonendstopped simple cells can appear endstopped. and the ES model will respond best to shorter lengths than those at the optimal orientation. These results should be evident. so we will not consider them further here.

The final series of orientation experiments deals with orientation alignment in the endzone. Figure 3.4 shows the response of the even instance to an optimally-oriented bar in the RF center along with a bar of various orientations centered in one of the end 
zones. Peak inhibition occurs for the preferred orientation of the central RF. It should also be clear that moving the center of the inhibitory bar from a position collinear with the central RF would diminish the inhibition. A similar result holds for the odd-symmetric model. Orientation-tuning of the end zones is consistent with the findings of Hubel and Wiesel (1965) and Orban et al (1979b). and collinearity of maximal end inhibition with maximal central excitation. which follows from the way the simple components are aligned. is also consistent with what is known of the physiology (Orban. et al, 1979b).

One point remains. and that is the relation of the simulations to the reported population statistics of cortical orientation-tuning Note that although orientation-tunıng is broadened with the shorter stimulus, the endstopped cell still has narrower tuning than its simple components. This is inconsistent with the reported population averages (Kato et al, 1978). but there are several possible reasons for this discrepancy. One is that the average broader tuning of endstopped than nonendstopped simple neurons is attributable to the endstopped cells being synthesized from a simple sub-population with broader orientation characteristics than the population as a whole. A second factor concerns how well the convolution model of the simple RF captures the interactions between RF subregions. In length-tuning measurements. moving and flashed measurements may correspond because the stimulus at any time is largely within one subregion. Such is not the case with orientation-tuning. and it is less clear that interactions that determine velocity and directional response can be ignored. Thirdly. suppose that end inhibition does not originate from a single large RF but from independent, spatially-displaced RFs. The observed lengthtuning curves can also be replicated with such a model. but the orientation-tuning characteristics could be different. The reason is that each end zone sees a displaced stimulus and hence responds over a narrower range - hence failing to narrow the orientation-tuning of the ES cell. In any case. either more specific cortical knowledge. or a physiological model more detailed than one with static. spatial RFs. is required to resolve these issues. 


\subsection{Curvature}

Having examined the relationship between the length and orientation tuning properties of endstopped neurons and the endstopped simple model. we now consider curvature. This section differs from the previous ones in treating aspects of the response properties of visual neurons that are only beginning to be investigated. Therefore. rather than representing model verifications, the results and observations take the form of predictions.

\subsubsection{Differential Geometry and Endstopping}

Curvature. conventionally described as the rate of change of the curve's tangent direction. can also be thought of as the rate at which a curve moves away from its tangent line. It is this perspective that furnishes insight into the curvature-response of the ES model. First consider the expected response of a simple cell operator to a semi-circle aligned so that its tangent at mid-arc is both centered on the RF and parallel to its long axis. The response is weak to a highly-curved arc but increases with the radius of curvature. This is because more of the curve passes through the excitatory center and less through the antagonistic sidebands as curvature decreases. Changing the size of the RF changes the rate at which response grows with the radius. Therefore RFs of different size provide information about the way in which a curve departs from straightness. Since the ES model is constructed with aligned RFs of different size. it is natural to consider its response to curves.

\subsubsection{Response of the ES Model to Curved Lines and Edges}

A distinction between even and odd-symmetric cells is commonly made based on their responses to lines and edges. The distinction is re-evaluated here in examining the response of combinations of such cells to curved lines (thin curves) and edges (wide curves). 
3. The Endstopped Simple Cell Model

\subsubsection{Curved Lines}

Figure 3.5 shows the response to semi-circular arcs as a function of radius of curvature. The response of the even-symmetric instance of the model and that of its component cells is shown in Figure 3.5a. The result is a kind of bandpass curvatureresponse. Because of the even-symmetry of the model components. the response is the same for both signs of curvature. As was the case with length-tuning, the non-monotonic response results from the difference in growth rate of the small and large cell responses - for some range of values the small cell responds well but stimuli in this range are too curved to strongly activate the large cell. Different sizes and weights of the small and large cells will produce curvature-response curves with different peaks, breadths and asymptotes. Comparing Figures $3.2 \mathrm{a}$ and $3.5 \mathrm{a}$, note that the peak curvature response is about 70 percent of the peak response to a short isolated line. Figure $3.5 \mathrm{~b}$ illustrates the response to curves laterally shifted a variable number of pixels on the RF. The zero shift position is when the curve is centered on the RF as in Figure 3.5a. All the responses have approximately the same form; they differ principally in magnitude.

Figure 3.6 shows the response of the odd-symmetric instance of the model to arcs of the two signs of curvature. In Figure 3.6a, the response to arcs that curve toward the antagonistic RF region is shown. The curvature-response is much like that seen in Figure 3.5a - a broadly curvature-selective response. In this case. for some range of curvatures, the arc largely avoids the antagonistic RF region of the small cell but curves into the antagonistic RF region of the large cell, reducing its response and hence its inhibition. Figure 3.6c shows the response to arcs of both signs of curvature. expressed in terms of curvature rather than radius of curvature. The left half of the graph corresponds to 3.6a. The right half shows that there is virtually no response for the other sign of curvature. The reason is that a curve which avoids the antagonistic RF region as it leaves the RF produces a strong response in the large cell and hence strong inhibition. Figures 3.6b.d depict the resporise to curves at different positions on the RF. It is evident that the curvature sign selectivity is preserved. 


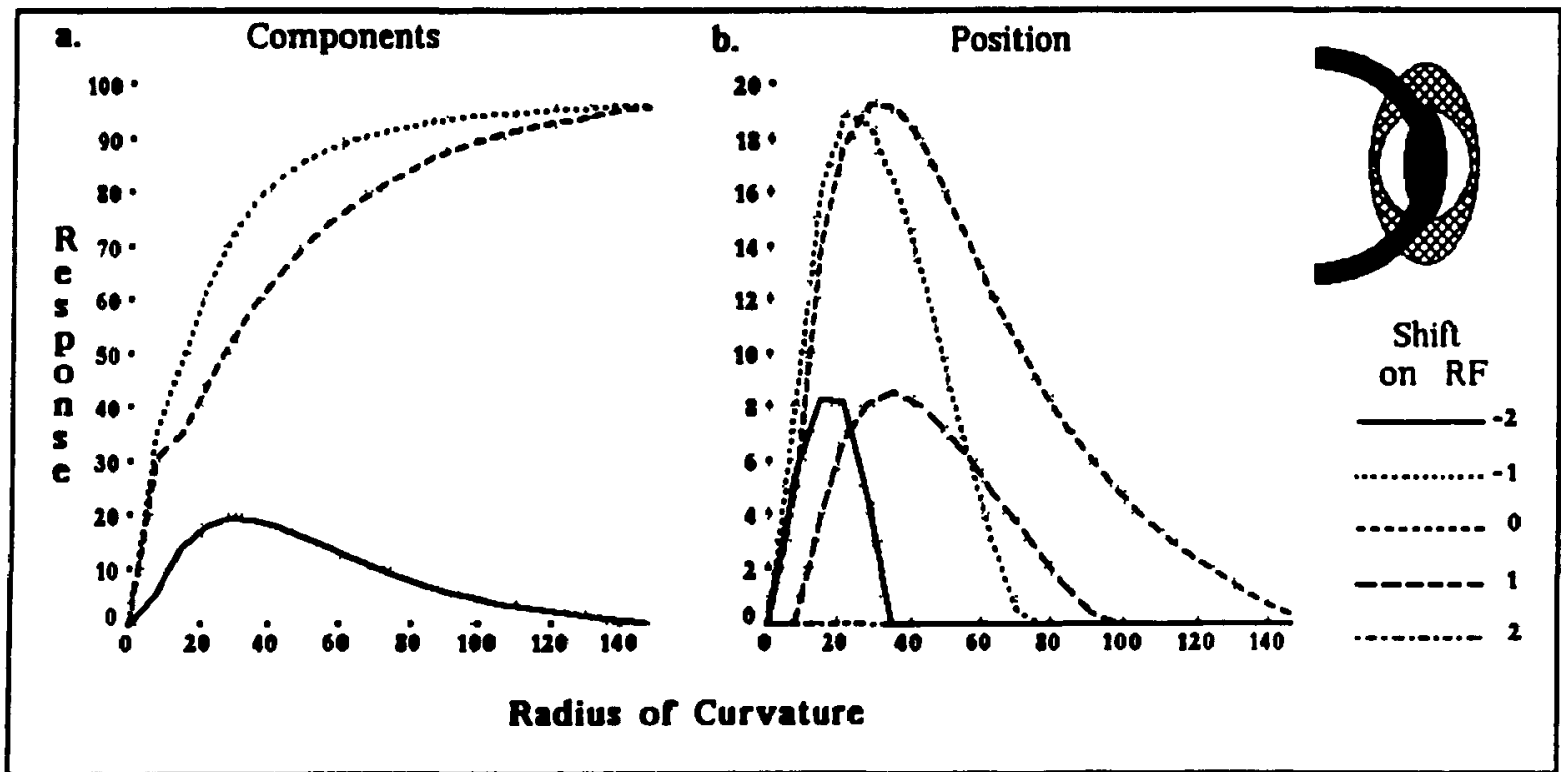

Figure 3.5 Curvature-tuning curves for an even RF ES cell. The parameters have the same values as in Figure 3.2n. The arcs are curved lines of width 3 pixels and vary in radius from 0 to 140 pixels in steps of 7 . (a). Response of the model cell and its components to one sign of curvature. The dotted and dashed lines are the small and large component responses. (b). Curvature response as a function of position across the RF. Negative and positive values represent left and right shifts. respectively, in the RF icon. There is no response at the +2 position.

For one sign of contrast the odd-symmetric cell model is selective for sign of curvature as well as magnitude. Reversing the curve contrast, however, reverses the curve sign preference. Therefore. taken in isolation, the response of this type of cell does not unambiguously signal curvature magnitude and sign. However, in combination with evensymmetric endstopped RFs or an odd-symmetric endstopped RF with positive and negative lobes reversed with respect to the first cell. both curve sign and contrast can be inferred.

A comparison of Figures $3.2 \mathrm{~b}$ and $3.6 \mathrm{a}$ shows that, unlike its even-symmetric counterpart. the odd-symmetric cell responds about 50 percent better to an optimallycurved stimulus than to a short optimal line. Instances of the model that respond more strongly to curves than short lines do so because a stimulus curving into the antagonistic RF region of the inhibitory cell elicits less inhibition than a short, isolated line.

\subsubsection{Curved Edges}

Figure 3.7 examines the response to curved edges. Parts a,b.c of the figure 

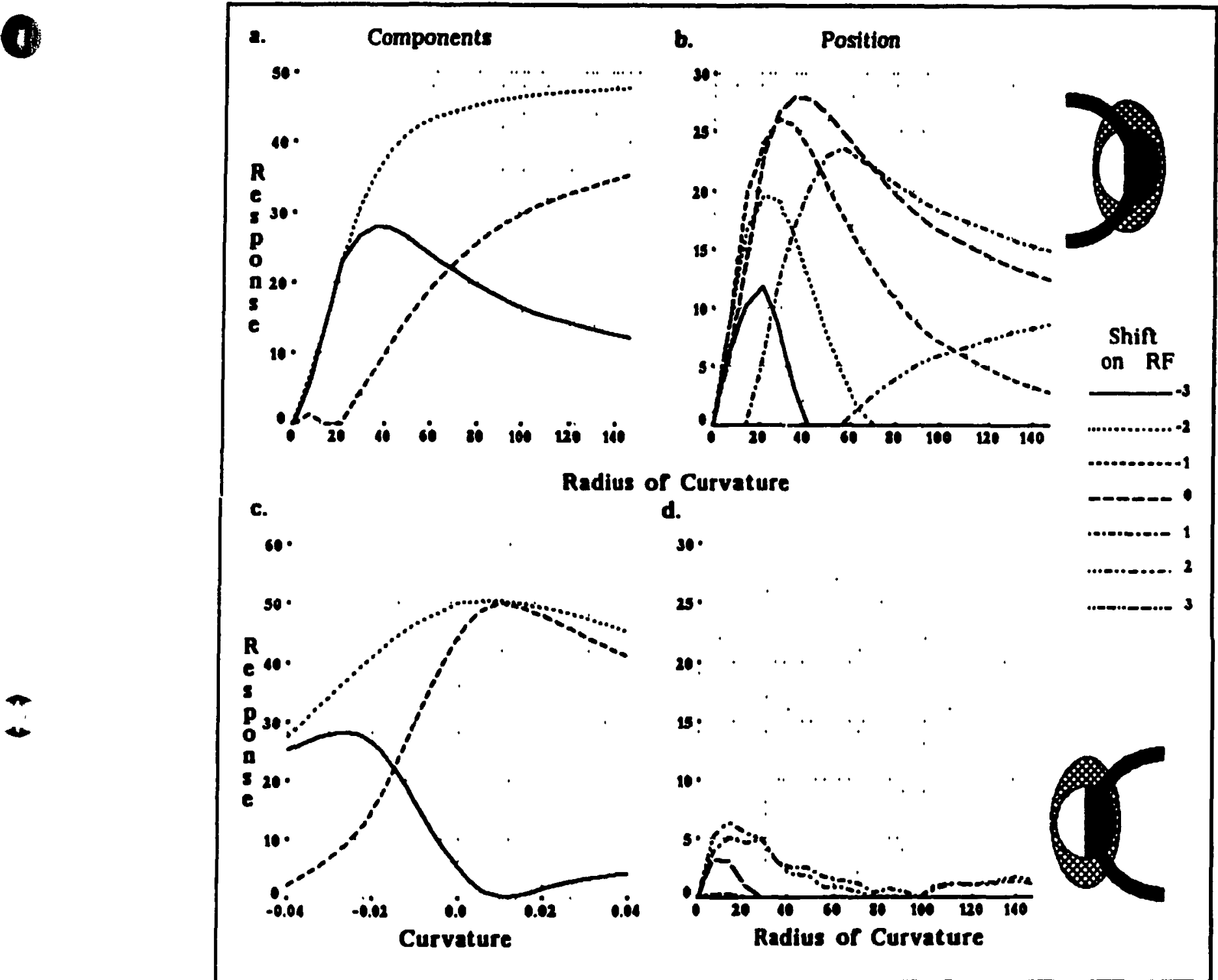

Figure 3.6 Curvature-tuning curves for an odd RF ES cell (same parameters as Figure $2 \mathrm{~b}$ ). The arcs are curved lines of width 3 pixels and vary in radius from 0 to 140 pixels in steps of 7 . (a). Response of the odd ES instance and its components to one sign of curvature (toward the antagonistic subregion). (b). Curvature response at 7 positions across the RF. The zero position represents the peak of the positive lobe of the small RF. (c). Cunvature response for both signs of curvature expressed in units of curvature (pizels ${ }^{-1}$ ). The left half of the graph corresponds to a. and the right half demonstrates the absence of response to the rightward curving arcs. (d). The response to rightward curving arcs as a function position on RF. Compare to b.

are obtained with an odd-symmetric instance having the same components as previously but with different relative weightings ( $c_{s}$ increased from 1.7 to 2.8 for balanced edge response). The response to an appropriately-oriented curved edge is shown in figure 3.7a. 
3. The Endstopped Simple Cell Model

The response is similar in form to those observed previously. The curved edge response at different positions is shown in $7 \mathrm{~b}$. Figure 3.7c shows the curvature response for both signs of curvature. maintaining the same edge contrast across the edge as the curvature goes through zero. The symmetry of the response to opposite contrasts in the two halves of the RF leads to the symmetric response There is no response to a curved edge of the opposite contrast

Figure 3.7d shows the combination of a small odd-symmetric RF with a large even-symmetric one. The cell that results from their convergence is selective for sign of curvature and contrast, independent of the magnitude of curvature. Hence this type would be useful in abstracting the curvature sign.

A summary of the response of the different model instances to curved edges and lines is given in Table 1 All the model instances examined are broadly-tuned for curvature magnitude. By employing a smaller excitatory cell or decreasing its weight, a shift in the peak of the curvature-tuning curve toward higher curvatures is effected. Alternatively. increasing the size of the inhibitory cell broadens curvature-tuning by shifting effective inhibition toward lower curvatures. ES instances composed of odd-symmetric components are selective for curvature sign for curved lines but not for curved edges while an ES cell with odd small component and even large component is selective for the sign of curvature of a curved edge. Since no single cell unambiguously signals both the magnitude and sign of curvature information independently of sign of contrast and whether the the stimulus is a line or edge, it follows that local network interactions between cells are necessary.

In our electrophysiological investigations of curvature-selectivity (Dobbins et al.. 1987) we found that different endstopped cells have different peaks and widths in curvaturetuning. and that some endstopped cells are selective for sign of curvature. However the mechanism of the sign-selectivity has not yet been investigated. In a related paper we examine how the choice of model parameter values affects curvature-response. its robustness under perturbations of the stimulus, and how the breadth of tuning determines the choice of a family of cells selective for different ranges of curvature (Dobbins et al, 1988). 


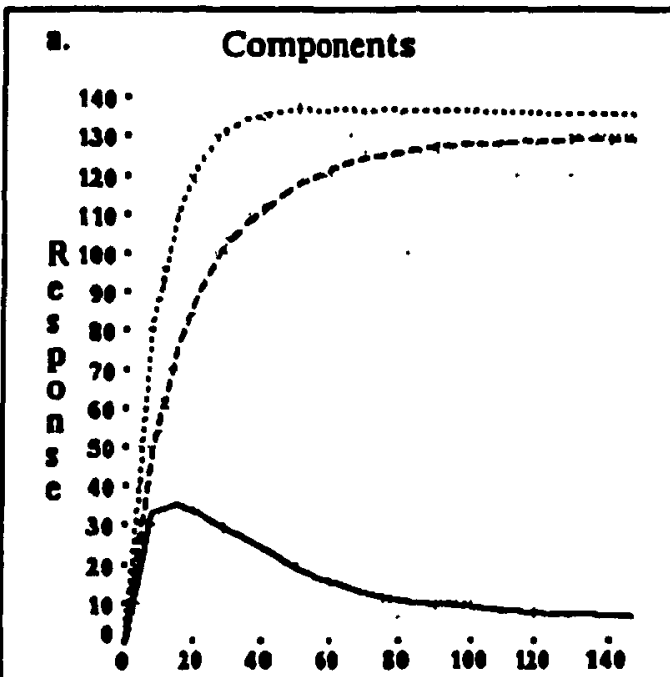

b.

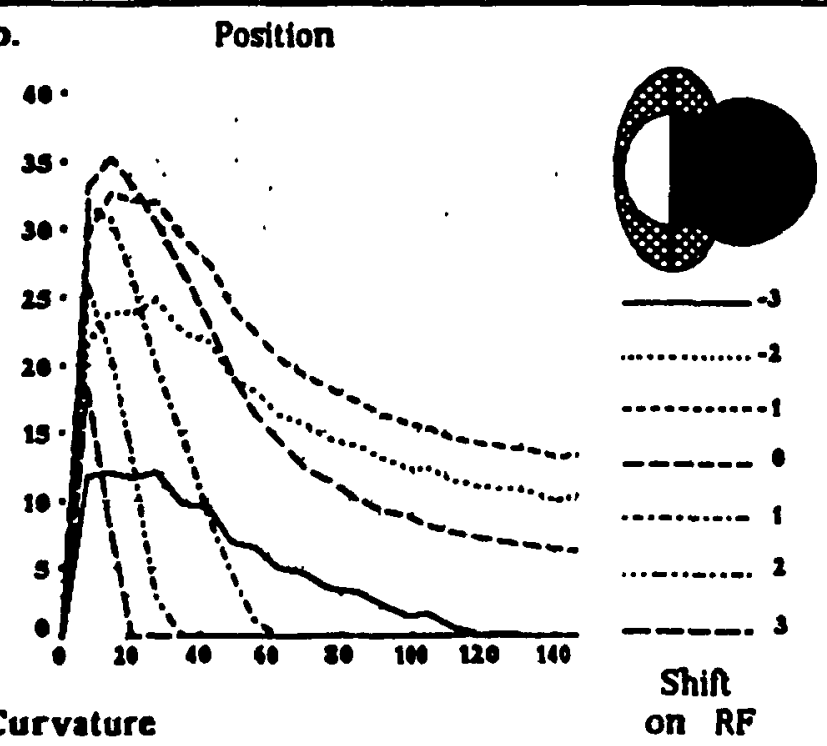

Radius of Curvature

on RF

c.

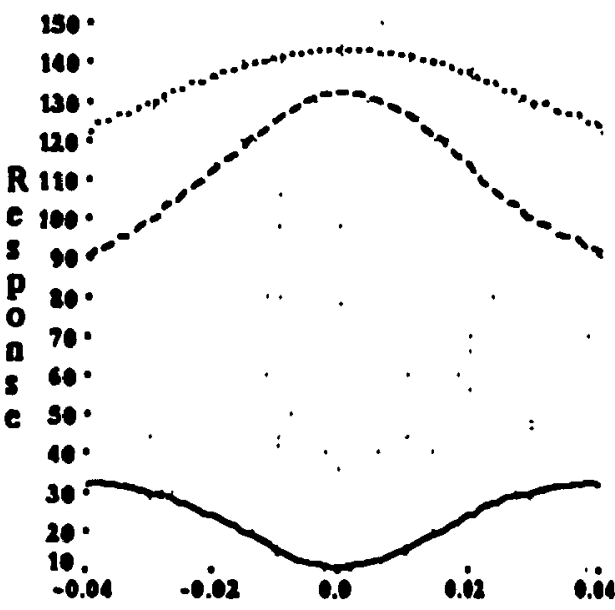

d.

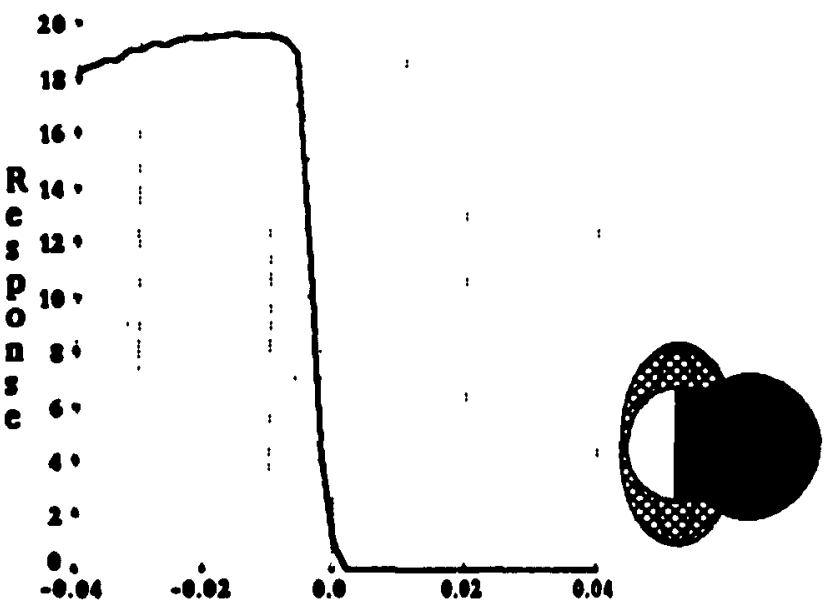

Curvature

Figure 3.7 Curvature-tuning curves for curved edges In a.b.c the odd model instance is used with $c_{s}$ increastd to 28 (from 17 in Figure $32 b$ and 36) (a) Response as a function of radius of curvature for one sign of curvature (b) Curvature response at 7 positions across the RF The zero position represents the vertical tangent to the edge aligning with the central division of the RF (c) Response as a function of curvature for both curve directions There is no response if the edge contrast is inverted. (f) Response of an ES instance with small odd and large even components and all parameters but the weights the same $\left(c_{S}=0.4\right.$ and $\left.c_{L}=1.0\right)$ The response depends on the sign of curvature but little on its magnitude.

\subsection{Response to Discontinuities and Singularities}

We now broaden the set of stimuli from idealized arcs and edges of constant 


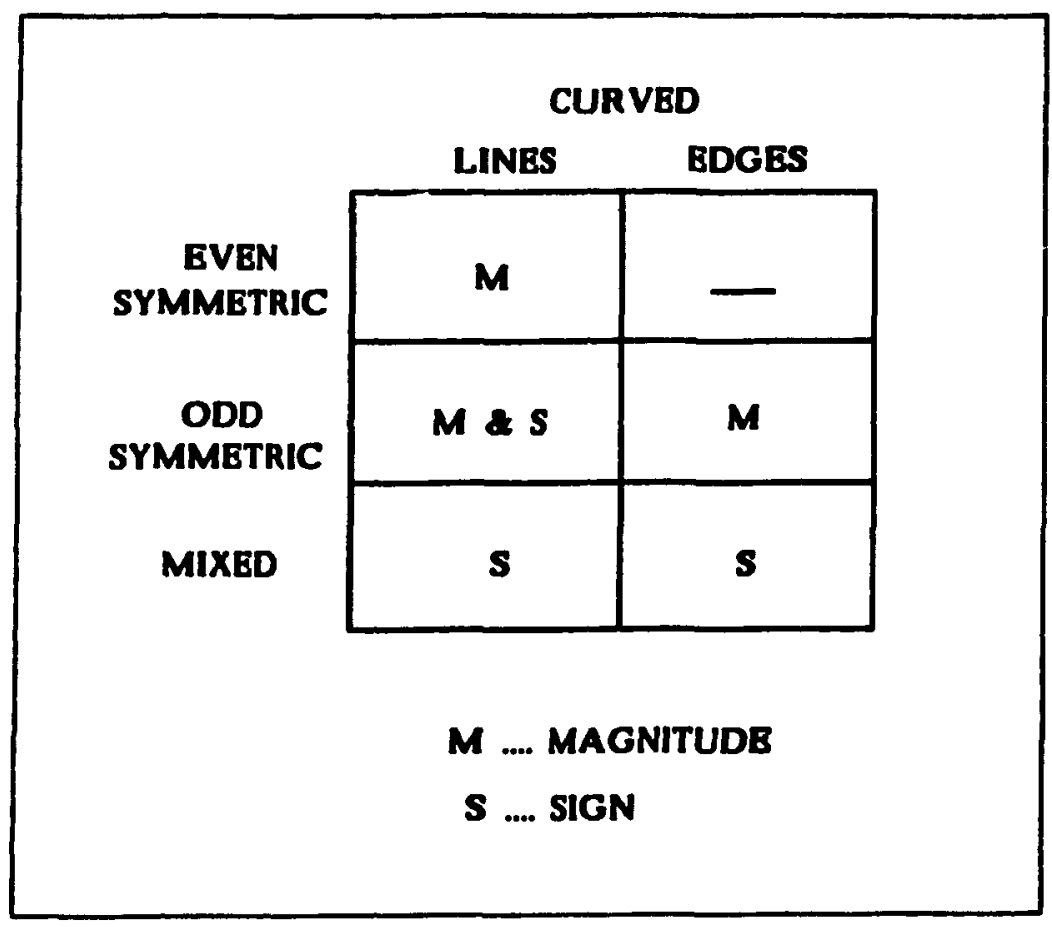

Table 3.1 ES Curvature Response Summary

curvature to a more general class of curves. Specifically. we consider the response to curves with (i) discontinuous first derivatives (chevrons) and (ii) sign changes in the second derivative (inflections).

\subsubsection{Response to Chevrons}

In Figure 3.8 is shown the response of the even-symmetric instance (of Figures 3.2a and 3.5) as a function of position along the stimulus shown. The model cell is horizontally-oriented and aligned with the horizontal line. The response never achieves one half the value obtained with an appropriate smooth curve or short, isolated line. The same is true if the short arm of the stimulus is rotated by $\mathbf{4 5}$ degrees to form a right angle. or by 90 degrees, or is removed. For such patterns the component responses remain balanced. and this prevents significant ES model response.

A second experiment involves varying the angle. and the response to such a stimulus is shown in Figure $3.9 \mathrm{a}$. The 180 degree position represents a straight line along the RF center. Near 180 degrees. as support for a central tangent increases. the components 


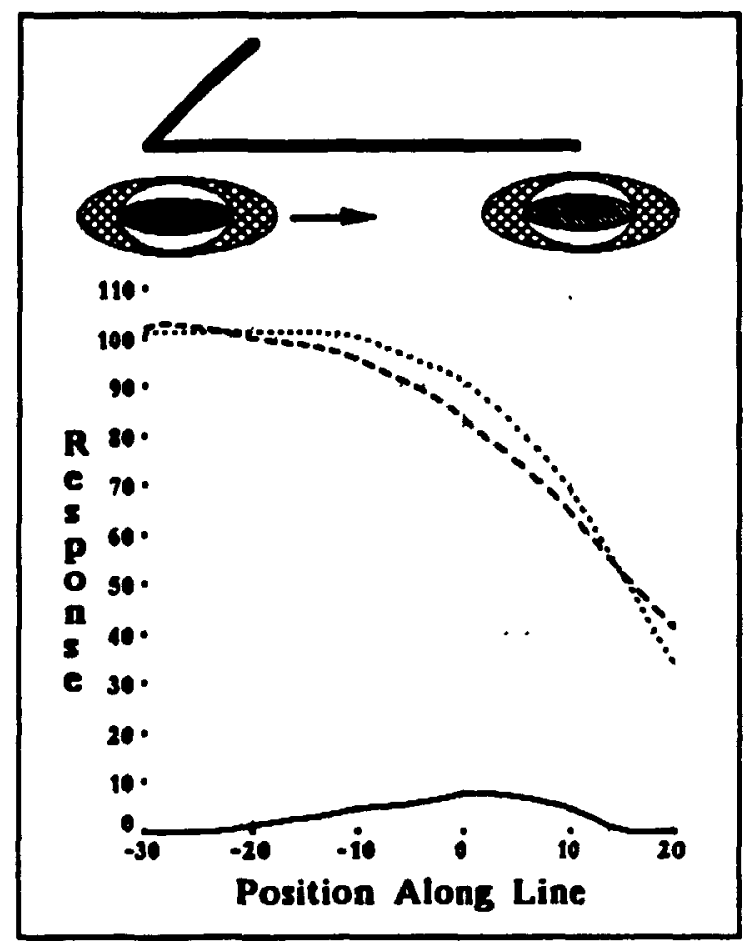

Figure 3.8 Response of even ES model as it moves along the stımulus shown (width 3 pixels) The response is not substantial at any position Simular results are found when the short arm of the stimulus has different orientations or is absent

respond strongly, but the balance between them prevents a substantial response from the ES cell. Figure $3.9 \mathrm{~b}$ shows the response as a function of orientation to a right angle with corner centered on the RF. The small and large components respond strongly when one arm of the stimulus aligns with the preferred orientation, but balance precludes a strong response

If. instead of an angled stimulus. a curved stimulus with a tangent discontinuity is employed. the response depends on the size of the discontinuity and its disposition with respect to the small excitatory RF as well as how the curve moves through the large component RF. If the line width is increased the stimulus floods the central excitatory RF and the response to chevrons and smooth curves are less distinct. However. larger endstopped cells tuned to lower spatial frequencies will now distinguish the stimuli. Together. the results shown in Figures 3.8 and 3.9 indicate that if the ES model has parameters chosen to elicit curvature-selective responses to smooth curves. angled and endline stimuli will be significantly less effective. and we would expect that doubly stopped neurons 


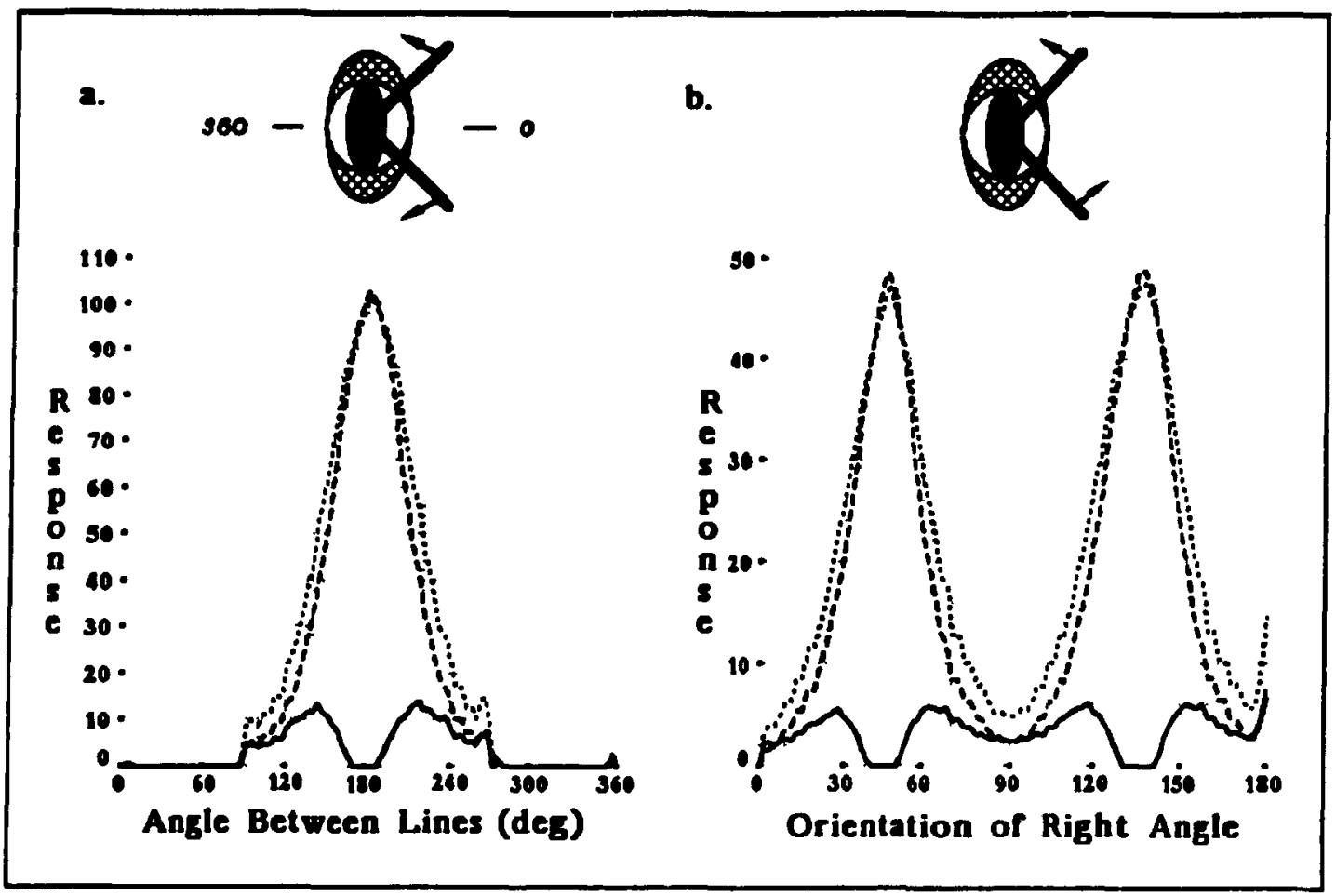

Figure 3.9 Response of the even ES instance to angled line stimuli (width 3 pixels) for different angles and orientations The solid lines depict the ES response. and the dotted and dashed lines the response of the small and large simple components. respectively (a) A variable angle stimulus of total length 60 pixels centered on the RF At 180 degrees the bars align along the RF long axis (b) A right angled stımulus centered on the RF is rotated In both experiments balance of the component responses lead to weak response from the ES cell

would not be strongly excited either The one qualification that should be made is that weakly endstopped cells (in the model obtained by decreasing the large cell gain. $c_{L}$ ) will be less stimulus-specific in general and curvature-selective in particular. That being said. we predict that endstopped neurons will respond differently to corners and smooth curves

\subsubsection{Response to Inflections}

There are two kinds of curvature changes: (i) changes in the magnitude and (ii) changes in the direction or sign. We now focus on the latter kind and Figure 3.10 shows the response to a curved stımulus with an inflection centered on the RF. The stimulus is discontinuous in curvature: there being an abrupt jump from constant curvature of one sign to the same magnitude and opposite sign at the center of the stimulus. However the tangent is well-defined at that point and aligns with the RF preferred orientation. The 
response of the even and odd versions of the model to inflections at different curvature magnitudes is shown in Figure 3.10a.b. Predictably, based on considerations of symmetry. the response of the even-symmetric cell is the same as for a stimulus that doesn't change sign of curvature (compare with Figure 3.5a) On the other hand. the response of the odd-symmetric operator to curved line inflections (Figure $310 \mathrm{~b}$ ) is approximately half way between the responses obtained to the two signs of curvature independently (Figure 36a.c) as would also be expected This points to a means of localizing inflections.

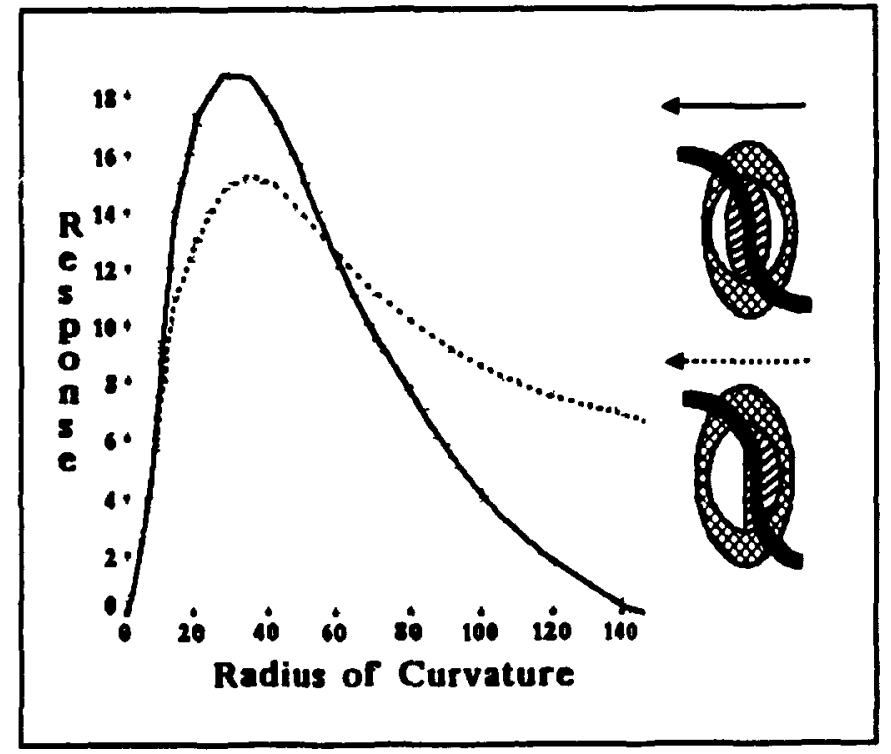

Figure 3.10 Response of even and odd ES models to inflections of a curved 3 line as a function of radius of curvature Parameter values as in Figure 2 The inflected stimulus (width 3 pixels) is obtained by reflecting half of a semi-circular arc about the tangent line at midarc The even ES instance (solid line) has the same response to the inflected stimuli as to a same-sign curve of the same radius The response of. the odd instance (dotted line) is half way between the responses obtained to the two signs of curvature - in most cases about half the response of the preferred sign stimulus

The responses of the model instances to edge inflections are not shown but can be understood based on symmetry. Since the odd-symmetric ES instance responds equally to both signs of curvature (provided the edge is of the right contrast sign) it will respond well to a stimulus which curves one way in one half of the RF and the other way in the other half. The mixed component model (of Figure 3.6c). the response of which depends critically on sign of curvature. gives a half response to the inflected edge stimulus. 


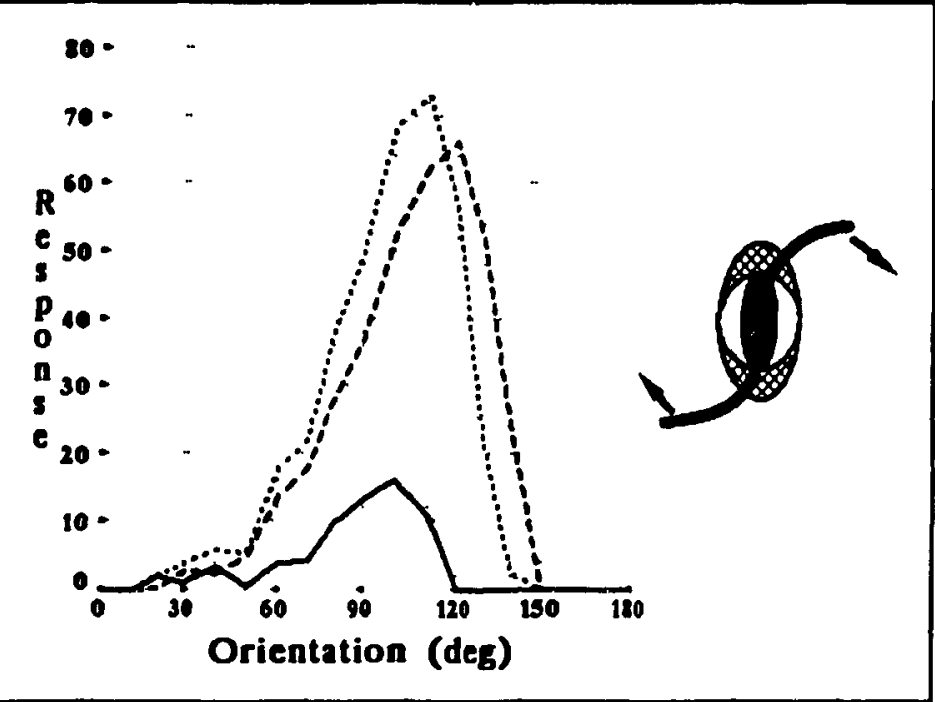

Figure 3.11 The response of the even ES instance and its components to a centrally. inflected cubic curve of width 3 pixels (and $k=0.003$ ) as a function of RF orientation All three responses have peaks shifted from the central tangent orientation (90 degrees) but the shift is least for the ES instance

In a third experıment we examined the response of the even ES instance and its components to an inflected curve as a function of curve orientation. The curve is of the form $y=k x^{3}$. where $k$ is chosen so that the the ES instance responds well. Figure 311 shows the response of the ES instance and its components to the curve as a function of orientation. Note that the peaks of all thr se responses are shifted from the orientation of the curve's central tangent. The shift is greater for the large simple cell than the small one and least for the ES instance. For yet larger simple RFs the peak would be even more shifted as the orientation that best matches a larger piece of the curve moves away from the tangent orientation The response of the ES cell is shifted least, precisely because of the difference in response shift for the small and large cells. From the point of view of the endstopped cell the inhibition is asymmetric with respect to the peak orientation of the small cell. therefore its peak response is closer to the tangent orientation than either component. This example illustrates how the tangent-curvature pair signaled by the ES model represents the local behaviour of the curve with greater fidelity than its linear components. 
We have shown that one way of thinking about the difference between nonendstopped and endstopped cells is in the way they respond to curves. Our model of endstopped simple neurons is length-selective in precisely the way that endstopped neurons are. It is also curvature-selective for arcs of the appropriate position and orientation. There are two crucial points to be emphasized: (i) the bandpass curvature response results from the difference of two low pass responses of different bandwidth, and (ii) the curvature sign-selectivity is determined simply from the RF symmetry.

Combining linear operators nonlinearly has led to a neuron model with specific response characterıstics. However, a number of simplifying assumptions have been made, especially regarding the spatial linearity implied by usıng convolution. In particular. Schumer and Movshon (1984) have shown that striate simple cells behave linearly when the length and contrast of a sinewave grating are modulated However. Hammond and Mackay (1983. 1985). using blobs of reversed contrast in the interrogating stimuli. demonstrate strong. non-linear, suppressive interactions. While more investigation of these issues is required, it Is theoretically attractive to consider the linear result as being expressed provided conditions do not occur that veto it. Such models are currently under investigation (Iverson. 1988: Zucker et al, in press).

Spatial nonlinearity is found in complex cells as well, and in neurophysiological experiments we have found that both simple and complex endstopped cells are curvature 
selective (Dobbins et al. 1987). Since the principal requirement for this class of model is orientation-selective end-inhibition. there is no reason to suppose that curvature selectivity in endstopped complex cells has a fundamentally different basis, and our initial explorations support this conjecture (Dobbins et al, 1988).

Another simplification is the use of half-wave rectification, but in other simulations we have found that substituting a smooth. saturating nonlinearity does not affect the results described here.

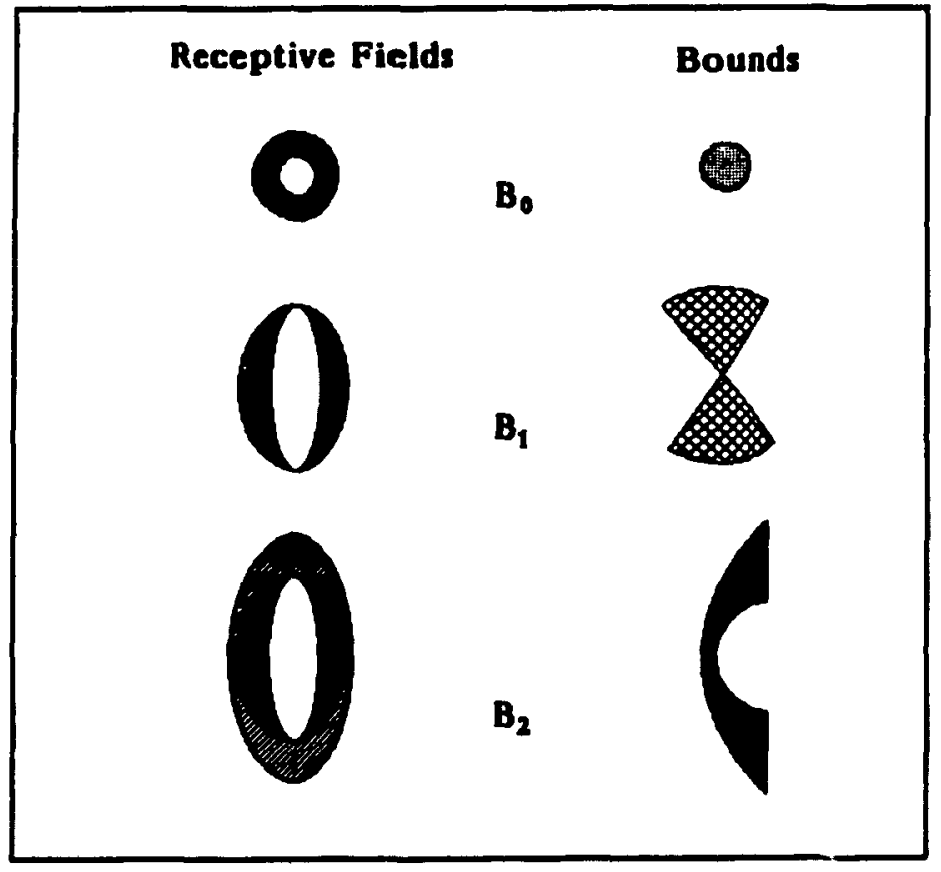

Figure 4.1 Illustration of how different classes of cells represent bounds on the derivatives of the contrast information The schematically represented retinal ganglion, nonendstopped and endstopped cortical neurons represent bounds on position $\left(B_{0}\right)$ : position and tangent $\left(B_{1}\right)$, and position, tangent and curvature $\left(B_{2}\right)$

Figure 4.1 illustrates a way of viewing the abstraction of spatial information as one proceeds from retinal ganglion and LGN neurons to nonendstopped and endstopped cortical neurons. Retinal ganglion RFs entail positional bounds $\left(B_{0}\right)$ on the contrast information they transmit, nonendstopped neurons an additional bound $\left(B_{1}\right)$ on tangent or orientation information. and endstopped neurons a further bound $\left(B_{2}\right)$ on curvature. Hence. endstopped neurons can be viewed as estimators of the $k$-jets of curves, $k \leq 4$ (Poston \& Stewart. 1978: Bruce \& Giblin. 1984) with the derivatives only known within inequality 
bounds. Koenderink and van Doorn have advanced the $k$-jet point of view (Koenderink, 1984: Koenderink \& van Doorn. 1986: Koenderink \& van Doorn. 1987). and given an example of a curvature operator that is similar to the endstopped simple neuron model we have developed but without the nonlinearity.

Note that endstopped cells do not estimate curvature in the sense of continuous mathematics. that is. as a limit tending to zero one example of this was provided by Figure 3.11: the central curvature of the cubic curve being zero. Rather, endstopped cells provide estimates of how a curve varies in a neighbourhood determined by the RF size. Consider a set of simple cells of various sizes and orientations centered at a point. Suppose that, for a particular orientation and in going from smaller to larger RFs, the responses are strong and then diminish. Most of the information resides in the RF size range over which the response transition occurs. and that information can be compactly represented in the responses of endstopped cells. Hence. from the $k$-jet perspective, endstopped cells can be viewed in two ways: the first as providing information about the spatial range over which a linear (simple cell-based) estimate is viable, and second as carrying the (tangent. curvature) estimate.

While endstopped neurons can provide orıentation and curvature information. it must be stressed that the tunıng curves are relatively broad. Some other processing is required to refine the estimates, to reduce unstructured noise responses, and to prevent inappropriate smoothing or interpolation between distinct image structures. We suspect network interactions play this role. and a variety of evidence supports lateral interactions that extend beyond the RF (for a review see: Allman et al., 1985). One would then expect that, in addition to the facilitatory interactions between collinear, co-oriented simple RFs reported by Nelson and Frost (1985). there should be interactions between endstopped cells which are tangent to a common circle (co-circular). Some of these constraints are being explored in computational models of network interactions underlying orientation selection (Parent \& Zucker. in press: Iverson. 1988: Zucker et al. in press). One conclusion seems clear, however: curvature is a key component in these models, and endstopped neurons are a sufficerit substrate for encoding it. 


\subsection{References}

Allman. J.. Miezin. F.. \& McGuinness (1985) Stimulus specific responses from beyond the classical receptive field: Neurophysiological mechanisms for local-global comparisons in visual neurons. Annual Rev. Neurosci. 8: 407-430.

Asada. H. \& Brady. M. (1986) The curvature primal sketch. IEEE Trans. P.A.M.I.. 8: 2-14.

Attneave. F. (1954) Some informational aspects of visual perception. Psychol. Rev.. 61. 183-193.

Blakemore. C.B. \& Over. (1974) Curvature detectors in human vision? Perception. 3: 3-7.

Bolz. J. \& Gilbert. C.D. (1986) Generation of end-inhibition in the visual cortex via interlaminar connections. Nature. 320: 362-365.

Bruce. J.W. \& Giblin. P.J. (1984) Curves and singularities. Cambridge Univ. Press. Cambridge.

Dobbins. A., Zucker. S.W.. \& Cynader. M.S. (1987) Endstopping in the visual cortex as a substrate for calculating curvature. Nature, 329. 438-441.

Dobbins. A.. Zucker. S.W.. \& Cynader, M.S. (1988) Designing endstopped neurons for curvature selectivity. TR-CIM-88-4. McGill University. Montreal.

Dreher. B. (1972) Hypercomplex cells in the cat's striate cortex. Invest. Opthalm. 11: 355-356.

Freeman. H. \& Davis. L.S. (1977) A cornerfinding algorithm for chain-coded curves. IEEE Trans. Comp., C-26: 297-303. 
Gibson. J.J. (1951) The perception of the visual world. Houghton. Mifflin.. Boston.

Gilbert. C.D. (1977) Laminar differences in receptive field properties of cells in cat primary visual cortex. J. Physıol. London. 268: 391-421.

Hammond. P. \& Andrews, D.P. (1978) Collinearity Tolerance of Cells in Areas 17 and 18 of the Cat's Visual Cortex: Relative Sensitivity to Straight Lines and Chevrons. Exp. Brain Res. 31: 329-339

Hammond, P. \& NacKay, D.M. (1983) Influence of lumınance gradient reversal on sımple cells in feline striate cortex. J. Physiol. London. 337: 69-87.

Hammond. P. \& MacKay. D.M. (1985) Influence of luminance gradient reversal on complex cells in feline striate cortex. J. Physiol. London. 359: 315-329

Hawken. M.J. \& Parker, A.J. (1987) Spatial properties of neurons in the monkey striate cortex. Proc. R. Soc. London Series B. 231 - 251-288.

Heggelund. P. (1986) Quantitative studies of the discharge fields of single cells in cat striate cortex. J. Physiol. London 373 277-292.

Heggelund. P. \& Hohmann. A. (1975) Responses of striate cortical cells to moving edges of different curvatures. Exp. Brain Res. 23: 211-216.

Hoffman, D.D. \& Richards. W. (1985) Parts of recognition. Cognition, 18: 65-96.

Hubel. D.H., \& Wiesel. T.N. (1962) Receptive fields. binocular interaction and functional architecture in the cat's visual cortex. J. Physiol. London. 160: 106-154.

Hubel. D.H.. \& Wiesel. T.N. (1965) Receptive fields and functional architecture in two non-striate visual areas (18 and 19) of the cat. J. Neurophysiol. 28: 229-89. 
Iverson. L.A. (1988) The Decription of Image Curves: Discrete Forms of Continuity in Space M. Eng. Thesıs. McGill University.

Jones. J.P \& Palmer. L.A (1987a) The two-dimensional spatial structure of simple receptive fields in cat striate cortex. J Neurophysiol., 58: 1187-1211

Jones, J.P. \& Palmer, L A (1987b) An evaluation of the two-dimensional Gabor filter model of simple receptive fields in cat striate cortex J. Neurophysiol., 58: 1233-1258.

Kato. H. Bishop. P.O.. \& Orban. G.A. (1978) Hypercomplex and simple/complex cell classifications in cat striate cortex. J. Neurophysiol., 41: 1071-95.

Koenderink. J J. \& Doorn. A.J. van (1976) The singularities of the visual mapping. Biol. Cybernetics. 24: 51-59.

Koenderınk J.J. (1984) The structure of images. Biol. Cybernetics, 50: 363-370.

Koenderink. J.J. \& Doorn. A.J. van (1986) Dynamic Shape. Biol. Cybernetics, 53: 383396.

Koenderink. J.J. \& Doorn. A.J. van (1987) Representation of local geometry in the visual system. Biol. Cybernetics, 55: 367-375.

Lehky. S.R. \& Sejnowski. T.J. (1988) Network model of shape-from-shading: neural function arises from both receptive and projective fields. Nature. 333: 452-454.

Leyton. M. (1988) A process-grammar for shape. Artificial Intelligence. 34: 213-247.

Marcelja. S. (1980) Mathematical description of the responses of simple cortical cells. J. Opt. Soc. Am., 70: 1297-1300. 
McGuire, B.A., Hornung. J-P., Gilbert, C.D.. \& Wiesel, T.N. (1984) Patterns of synaptic input to layer 4 of cat striate cortex. I Neurosci.. 4: 3021-33.

Movshon. J.A.. Thompson. I.D., \& Tolhurst. D.J. (1978) Spatial summation in the receptive fields of simple cells in the cat striate cortex J. Physiol. London. 283. 53-77.

Nelson. J.J. \& Frost. B.J. (1985) Intracortical facilitation among co-oriented. co-axially aligned simple cells in cat striate cortex. Exp. Br. Res.. 61. 54-61.

Orban. G.A. (1984) Neuronal operations in the visual cortex. Springer. Berlin

Orban. G.A.. Kato. H., \& Bishop. P.O. (1979a) End-zone region in receptive fields of hypercomplex and other striate neurons in the cat. J. Neurophysiol.. 42. 818-32.

Orban. G A.. Kato. H.. Bishop. P.O. (1979b) Dimensions and properties of end-zone inhibitory areas in receptive fields of hypercomplex cells in cat striate cortex. J. Neurophysiol.. 42: 833-49.

Orban. G.A.. Versarel. M.. \& Lagae. L. (1987) How do striate neurons represent curved stimuli? Soc. Neurosci. Abstr. XIII 404.10. New Orleans.

Parent. P.. \& Zucker, S.W. (1988) Trace inference, curvature consistency. and curve detection. IEEE Trans. P.A.M.I. (accepted for publication).

Parker, A.J. \& Hawken, M.J. (1988) Two-dimensional spatial structure of receptive fields in monkey striate cortex. J.O.S.A. Series A. 5: 598-605.

Poston. T. \& Stewart. I.N. (1978) Catastrophe theory and its applications. Pitman. London.

Rose. D. (1977) Responses ot single units in cat visual cortex to moving bars of light as a function of bar length. J. Physiol. London London 271: 1-23. 
Schumer, R.A. \& Movshon, J.A. (1984) Length summation in simple cells of cat striate cortex. Vision Res. 24: 565-571.

Timney. B.T.. \& Macdonald. C. (1978). Are curves detected by 'curvature detectors'? Perception. 7: 51-64.

Zucker. S.W. (1986) The computational connection in vision: early orientation selection. Behav. Res. Meth., Instr., and Computers. 18: 608-617.

Zucker, S W.. Dobbins, A , \& Iverson, L (1989). Two stages of curve detection suggest two styles of neural computation. Neural Computation. 1 accepted for publication. 


\section{Appendix A. Appendix 1}

In Chapter 3 it was shown that for particular choices of the parameter values. the ES model responds in a curvature-selective manner. In this section the factors affecting parameter choice are briefly examıned.

The gain of the components $\left(c_{S}, c_{L}\right)$ determines the strength of the end inhibition. For $c_{S}, c_{L}$ large, the model becomes less endstopped. Correspondingly, the rolloff rate of curvature response on the low curvature side decreases. Hence. the range of curvatures to which the cell responds increases Conversely. decreasing $c_{S}, c_{L}$ increases endstopping and curvature specificity, and decreases response gain

Since curvature is the rate of change of orientation with respect to arc length. orientation tuning has a significant effect on curvature response. Orientation bandwidth is principally determined by two parameters: RF length $\left(4 \sigma_{y}\right)$ and the peak spatial frequency $\left(\omega_{0}\right)$ or equivalently. spatial wavelength $\left(\lambda_{0}=2 \pi / \omega_{0}\right)$ across the RF In the spatial domain

$$
B W_{G}=k \frac{\sigma y}{\lambda_{0}}
$$

where $k$ is a proportionality constant. Ideally. the small and large ES components would be matched in both orientation bandwidth and spatial frequency (peak and bandwidth) In that way the components would exhibit matched response variation to perturbations of curve orientation or spatial frequency content. However since the RFs are of different length. Equation A1 implies that the two conditions cannot be simultaneously satisfied. In

Dobbins et al (1988) this problem is treated at length. The parameter values in the two components represent a compromise between matching spatial frequency and orientation tuning. 